\title{
The economic burden of patient safety targets in acute care: a systematic review
}

\author{
This article was published in the following Dove Press journal: \\ Drug, Healthcare and Patient Safety \\ 4 October 2012 \\ Number of times this article has been viewed
}

\section{Nicole Mittmann' \\ Marika Koo' \\ Nick Daneman² \\ Andrew McDonald ${ }^{3}$ \\ Michael Baker ${ }^{4}$ \\ Anne Matlow ${ }^{5}$ \\ Murray Krahn ${ }^{6}$ \\ Kaveh G Shojania ${ }^{7}$ \\ Edward Etchells ${ }^{7}$}

'Health Outcomes and Pharmaco Economics (HOPE) Research Centre, Division of Clinical Pharmacology,

2Division of Infectious Diseases, ${ }^{3}$ Quality and Patient Safety,

Sunnybrook Health Sciences Centre, Toronto, ON, Canada; ${ }^{4}$ Patient Safety in Ontario, University Health Network, Toronto, ON, Canada; ${ }^{5}$ Infection Prevention and Control and Patient Safety, Hospital for Sick Children, Toronto, ON, Canada;

${ }^{6}$ Toronto Health Economics and Technology Assessment (THETA) Collaborative, University of Toronto, Toronto, ON, Canada; ${ }^{7}$ University of Toronto Centre for Patient Safety, Sunnybrook Health Sciences Centre, Toronto, ON, Canada

Correspondence: Nicole Mittmann 2075 Bayview Ave E240, Toronto, ON, Canada M4N 3M5

$\mathrm{Tel}+\mathrm{I} 4164806100$ ext I652

Fax + I 4164806025

Email nicole.mittmann@sunnybrook.ca
Background: Our objective was to determine the quality of literature in costing of the economic burden of patient safety.

Methods: We selected 15 types of patient safety targets for our systematic review. We searched the literature published between 2000 and 2010 using the following terms: "costs and cost analysis," "cost-effectiveness," "cost," and "financial management, hospital." We appraised the methodologic quality of potentially relevant studies using standard economic methods. We recorded results in the original currency, adjusted for inflation, and then converted to 2010 US dollars for comparative purposes $(2010$ US $\$ 1.00=2010 € 0.76)$. The quality of each costing study per patient safety target was also evaluated.

Results: We screened 1948 abstracts, and identified 158 potentially eligible studies, of which only $61(39 \%)$ reported any costing methodology. In these 61 studies, we found wide estimates of the attributable costs of patient safety events ranging from $\$ 2830$ to $\$ 10,074$. In general hospital populations, the cost per case of hospital-acquired infection ranged from $\$ 2132$ to $\$ 15,018$. Nosocomial bloodstream infection was associated with costs ranging from $\$ 2604$ to $\$ 22,414$. Conclusion: There are wide variations in the estimates of economic burden due to differences in study methods and methodologic quality. Greater attention to methodologic standards for economic evaluations in patient safety is needed.

Keywords: patient safety, burden of illness, review, quality

\section{Introduction}

Patient safety has received considerable public, professional, political, and scientific attention over the past decade. Although the human burden associated with adverse events is well established, the economic cost of patient safety has received less attention. Despite the substantial effort that has been expended to develop and implement safety improvements, there is uncertainty about both the economic impact of unsafe care and the improvement strategies that offer the best value. Significant resources have been expended across the world to reduce patient safety events through interventions, without clear improvements. ${ }^{1,2}$

A fuller understanding of the economic burden of patient safety may inform health policy, health services research priorities, safety improvement priorities, and patient safety priorities. High quality data on economic burden of a condition are an essential component of comparative economic analyses such as cost-effectiveness analyses.

The objective of an economic burden study is to describe the economic impact of a patient safety target. These types of studies generally examine the overall cost of the condition to an environment (eg, acute care setting, society). 
Economic burden studies should be based on rigorous analytical methods, be impartial and credible in the use of data, and be transparent for and accessible by the reader. ${ }^{3}$ Economic burden studies are conducted using recognized frameworks which can be modified for specific target conditions. ${ }^{4,5}$ Drummond and Jefferson and Drummond et al constructed a checklist of economic parameters used worldwide. ${ }^{6,7}$ Economic burden studies should clearly outline the resource studies, the method for attributing costs to these resources, the method for measuring the resources used, the time frame for measuring the resources, and the economic perspective (hospital, third-party payer, or society) from which the resources were measured.

Our objective was to determine the quality of literature in costing of the economic burden of patient safety targets in the acute care environment.

\section{Methods}

We developed a list of patient safety targets based on prior systematic reviews, ${ }^{8}$ and existing national and international safety initiatives. ${ }^{9,10}$ Patient safety targets were based on three characteristics: (1) a clinical outcome (eg, hospital-acquired methicillin-resistant Staphylococcus aureus [MRSA] infection) or a surrogate with an established link to a clinical outcome (eg, MRSA colonization); (2) high specificity as a measure of patient safety, as opposed to being a naturally occurring condition; and (3) a sufficiently long history of measuring this outcome in the literature, such that some studies on the economic burden could be expected.

Patient safety targets included: adverse events, adverse drug events, ventilator-associated pneumonia, nosocomial urinary tract infection, antibiotic-resistant organism colonization, antibiotic-resistant organism infection, catheterassociated bloodstream infection, nosocomial Clostridium difficile-associated disease, surgical site infection, nosocomial pressure ulcers, wrong site surgery, retained surgical foreign bodies, contrast-induced nephropathy, nosocomial venous thromboembolism, and nosocomial fall-related injuries. We also included six improvement strategies (hand hygiene, rapid response teams, bundles, check-lists, automatic stop orders and bar coding) to ensure that we obtained all relevant economic literature that may not be captured through searches based solely on patient safety targets.

We sought burden-of-illness or cost-of-illness studies. A search was performed using the MEDLINE database for articles published between 2000 and 2010 using the following search terms for costs: "costs and cost analysis" (Medical Subject Headings [MeSH]), "cost-effectiveness" (text word), "cost" (text word), and "financial management, hospital" (MeSH). We also searched the Agency for Healthcare Research and Quality Patient Safety Network (see http:// psnet.ahrq.gov) using the term "cost."

Reviews, editorials, and articles with no costing information in the abstract were excluded. One member of the study team (MK) excluded reviews, editorials, and articles with no costing information in the abstract. Two independent members of the study team (MK and EE) reviewed the remaining abstracts and obtained the full publication of any abstract considered potentially relevant by either member. Full publications of any abstracts considered potentially relevant were retrieved. Two investigators (EE and NM) independently evaluated each publication, using adapted relevant methodologic features $(\mathrm{n}=21)$ as described by Drummond and Jefferson. ${ }^{6}$ Each feature was arbitrarily scored one point, for a maximum score of 21. Features from the original list were excluded if they were not applicable to economic burden studies. If the two reviewer scores were within five points of one another, the higher score was taken. Otherwise, reviewers met to discuss and resolve discrepancies.

We report all cost data in 2010 United States dollars for comparative purposes between patient safety targets. The original year and currency is stated in the summary data. Each cost was first converted to US dollars of the same year as indicated in the publication using the Bank of Canada currency converter. ${ }^{11}$ Then, each converted cost was inflated to 2010 US dollars using the United States Department of Labor Bureau of Labor Statistics inflation calculator. ${ }^{12}$

\section{Results}

Our initial search yielded 1948 citations. We screened out 1749 abstracts that were review articles, editorials, or lacking in any costing information or results. We obtained and reviewed the remaining 199 publications. We excluded 41 publications for the following reasons: no evaluation of the economic burden of a patient safety target $(n=5)$, no primary data on economic burden $(n=23)$, not conducted in an acute care setting $(n=7)$ or were review papers with no primary data or analysis $(n=6)$. This left 158 potentially eligible publications. Ninety-seven (61\%) of the 158 potentially eligible publications described no economic methodology despite reporting an estimate of economic burden and were excluded from further review (Figure 1).

\section{Methodologic quality}

For the remaining 61 studies, the median methodologic feature score was 15/21 (mean 14.6 \pm 21 , range 9-20). All studies had 


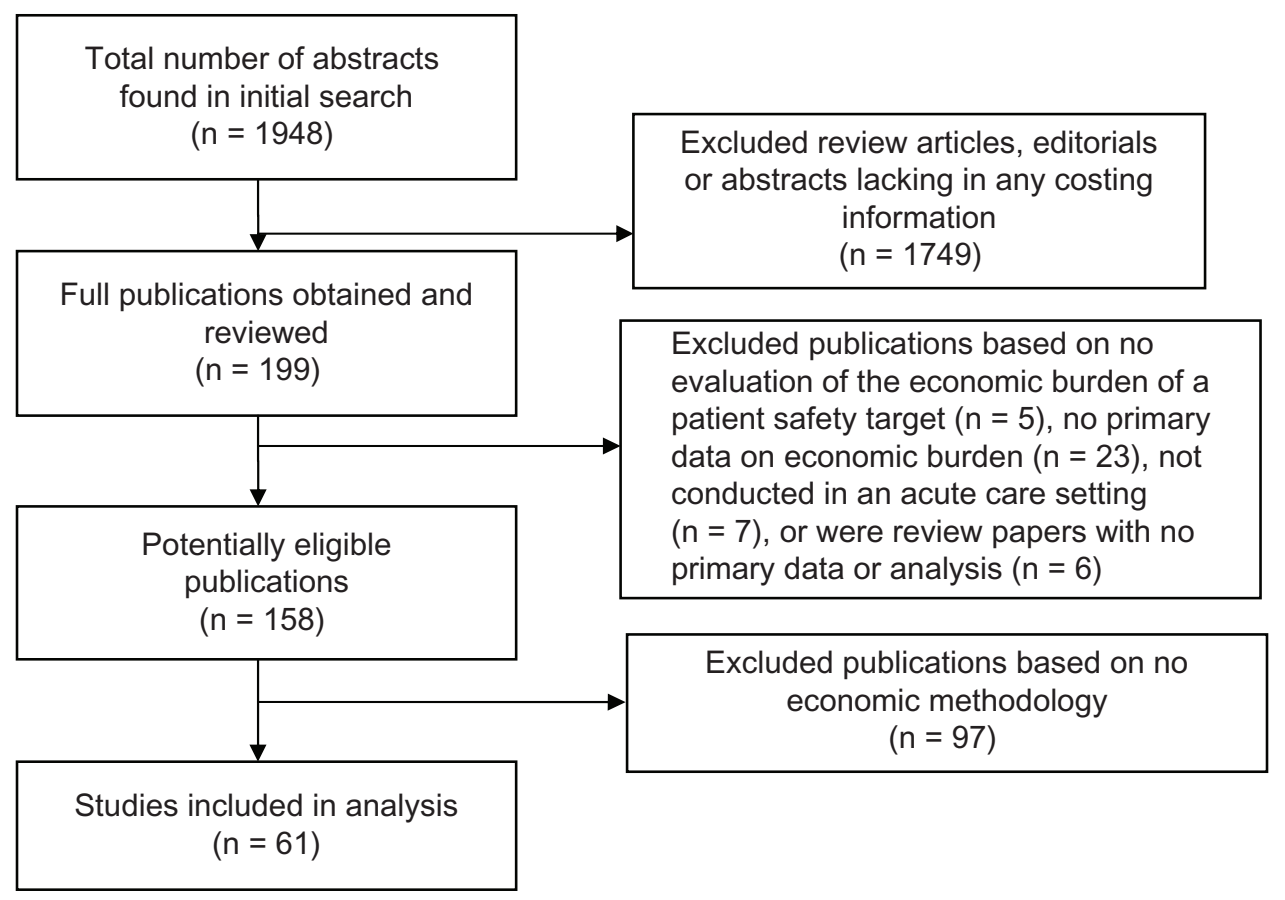

Figure I Results of screening and exclusion process.

essential methodologic features such as a statement of the research question, a statement of the economic importance of the research question, and a justification of the economic viewpoint. Fewer than $50 \%$ of these 61 studies reported productivity changes, discussed the relevance of productivity changes, provided details of inflation adjustments or currency conversions, or described any sensitivity analyses. Studies used different methods for identifying attributable cost, including propensity scores, case-controls, and regression analysis (Tables 1 and 2).

There was heterogeneity in study populations, resources incorporated, methods and results, so economic burden summary for all patient safety targets could not be calculated. We summarize the methodologic feature scores and range of results for each type of adverse event in Table 1. The maximum score was 21 . We provide detailed summaries of each study organized by patient safety target (Table 3 ).

\section{General studies of adverse events and adverse drug events}

We identified eight studies of the economic burden of adverse events and adverse drug events published since 2000. Five of these studies used a retrospective cohort study design, and relied on regression analyses to determine the attributable costs. Of these, two articles broadly focused on any adverse event or hospital-acquired complication. ${ }^{13,14}$ An additional article evaluated the economic burden of a broad range of adverse events in patients with spinal cord injuries. ${ }^{15}$ One article included five specific adverse events: medication errors, patient falls, urinary tract infection, pneumonia, and pressure ulcers. ${ }^{16}$ Another article evaluated costs related only to surgical adverse events, but did not further define them. ${ }^{17}$ The three remaining studies related to adverse events were either case series ${ }^{18}$ or prospective cohorts with nested cases and controls. ${ }^{19,20}$ Two of these studies defined a case as any adverse events ${ }^{19}$ or a case leading to a medical dispute. ${ }^{18}$ One study specifically evaluated adverse drug events. ${ }^{20}$

Costs attributable to adverse events were $\$ 4571^{19}$ and $\$ 10,074^{14}$ in two studies. In patients with spinal cord injury, the cost attributable to adverse events was $\$ 6258$, but were significantly higher for specific complications; for example, procedural complications in these patients were associated with additional costs of $\$ 20,183 .{ }^{15}$ The cost attributable to adverse drug events was $\$ 2830 .{ }^{20}$ In another study, medication error in medical and surgical cases were associated with costs of $\$ 361$ and $\$ 568$, respectively. ${ }^{16}$ Attributable length of stay related to adverse events ranged from 0.77 days to 32 days. ${ }^{15,19,20}$ Three of the eight articles did not record length-of-stay data. ${ }^{16-18}$

\section{Nosocomial infections (not otherwise specified)}

We identified ten studies of the economic burden of general nosocomial infections not otherwise specified by type 
Table I Summary of studies of economic burden of patient safety targets in acute care (detailed summary of each study is in Table 3)

\begin{tabular}{|c|c|c|c|}
\hline & $\begin{array}{l}\text { Number of } \\
\text { studies }\end{array}$ & $\begin{array}{l}\text { Mean methodologic feature } \\
\text { score [median, range] }\end{array}$ & Study design \\
\hline $\begin{array}{l}\text { Adverse events and } \\
\text { adverse drug events }\end{array}$ & 8 & $13[13,12-16]$ & $\begin{array}{l}\text { Retrospective cohort study }(n=5) \text {, } \\
\text { prospective cohorts with nested } \\
\text { cases and controls }(n=2) \text {, } \\
\text { case series }(n=I)\end{array}$ \\
\hline $\begin{array}{l}\text { Nosocomial infections } \\
\text { (not otherwise specified) }\end{array}$ & 10 & $14[15,12-16]$ & $\begin{array}{l}\text { Prospective study }(n=I) \text {, } \\
\text { retrospective cohort }(n=5) \text {, retrospective case } \\
\text { control study }(n=3) \text {, decision model }(n=I)\end{array}$ \\
\hline Surgical site infections & 8 & $14[|4.5| 1-,\mid 7]$ & $\begin{array}{l}\text { Prospective study }(n=I), \\
\text { retrospective cohort }(n=3) \text {, retrospective case } \\
\text { control study }(n=2) \text {, nested case control }(n=2)\end{array}$ \\
\hline $\begin{array}{l}\text { Nosocomial bloodstream } \\
\text { infections }\end{array}$ & 10 & $14[15,9-18]$ & $\begin{array}{l}\text { Prospective study }(n=I) \text {, } \\
\text { retrospective cohort }(n=3) \text {, retrospective case } \\
\text { control study }(n=5) \text {, case series }(n=1)\end{array}$ \\
\hline Nosocomial sepsis & 2 & $17[17.5,15-20]$ & $\begin{array}{l}\text { Prospective cohort }(n=I) \\
\text { retrospective cohort }(n=I)\end{array}$ \\
\hline $\begin{array}{l}\text { Nosocomial rotavirus } \\
\text { infections }\end{array}$ & 3 & $14[14,13-15]$ & $\begin{array}{l}\text { Prospective cohort }(n=I) \text {, } \\
\text { prospective case series }(n=I) \text {, } \\
\text { nested case control }(n=I)\end{array}$ \\
\hline $\begin{array}{l}\text { Nosocomial urinary } \\
\text { tract infection }\end{array}$ & 4 & $13[15,9-15]$ & $\begin{array}{l}\text { Prospective cohort }(n=I) \text {, } \\
\text { retrospective cohort }(n=2) \text {, } \\
\text { retrospective case control }(n=I)\end{array}$ \\
\hline Nosocomial pneumonia & 4 & $14[13-15]$ & $\begin{array}{l}\text { Prospective cohort }(n=2) \text {, prospective/ } \\
\text { retrospective case control }(n=I) \\
\text { retrospective case control }(n=I)\end{array}$ \\
\hline $\begin{array}{l}\text { Nosocomial respiratory } \\
\text { tract infection }\end{array}$ & 3 & $15[15,15-15]$ & $\begin{array}{l}\text { Retrospective cohort }(n=I) \\
\text { retrospective case control }(n=I) \\
\text { one case control }(n=I)\end{array}$ \\
\hline $\begin{array}{l}\text { Miscellaneous nosocomial } \\
\text { infections }\end{array}$ & 12 & $15[14.5,12-20]$ & $\begin{array}{l}\text { Prospective nested case control }(n=I) \text {, } \\
\text { case control }(n=I) \text {, } \\
\text { retrospective case series }(n=4) \text {, } \\
\text { retrospective case control }(n=2) \text {, } \\
\text { retrospective cohort }(n=3) \text {, retrospective } \\
\text { nested case control }(n=I)\end{array}$ \\
\hline $\begin{array}{l}\text { Nosocomial venous } \\
\text { thromboembolism }\end{array}$ & 2 & $17[17,16-18]$ & $\begin{array}{l}\text { Decision analysis }(n=I) \text {, retrospective } \\
\text { observational cohort study }(n=I)\end{array}$ \\
\hline Nosocomial falls & 3 & $15[15,14-16]$ & $\begin{array}{l}\text { Prospective cohort }(n=1) \\
\text { case series }(n=2)\end{array}$ \\
\hline Total & $68^{a}$ & & \\
\hline
\end{tabular}

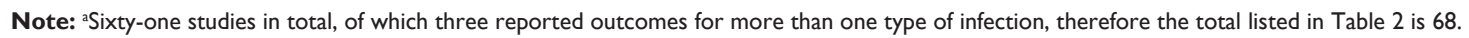

of infection. These included one prospective design, five retrospective cohort designs, three retrospective case control designs, and one decision model. Analytic methods included regression analysis, such as linear regression, multivariate regression, and ordinary least-squares regression analysis.

In general hospital populations, the cost per case of hospital-acquired infection ranged from $\$ 2132$ to $\$ 15,018 .^{21-27}$ Hospital-acquired infections cost \$2910 in gastrectomy patients $^{28}$ and $\$ 21,856^{29}$ in neonates. The estimated costs of hospital-acquired infections over one fiscal year in New Zealand in medical patients were US\$5,626,640 and surgical patients were US $\$ 4,803,046 .{ }^{30}$

\section{Surgical site infections}

We found eight studies of the economic burden of surgical site infections. Study designs included prospective cohort $(\mathrm{n}=1)$, retrospective cohorts $(n=3)$, retrospective case control $(n=2)$, and two nested case control designs. The average cost per case of surgical site infection in a general patient population was reported to be $\$ 1105,,^{22} \$ 2604,{ }^{31}$ and $\$ 14,422^{32}$ in three studies. In orthopedic patients, the median attributable cost of surgical site infection was $\$ 24,058 .{ }^{33}$ Surgical site infection in patients after colorectal procedures was $\$ 14,868,{ }^{34}$ head-andneck cancer-related surgery was $\$ 22,234,{ }^{35}$ coronary artery bypass graft procedures were $\$ 10,245,{ }^{36}$ and low transverse 
Table 2 Methodologic characteristics of studies of economic burden of patient safety events in acute care $(n=6 \mathrm{I})$

\begin{tabular}{ll}
\hline Methodologic feature & $\begin{array}{l}\text { Studies with this feature } \\
\text { present } \%(n)\end{array}$ \\
\hline
\end{tabular}

\section{Study design}

The research question is stated

$100 \%(61)$

The economic importance of the research question is stated

The viewpoint(s) of the analysis are

$100 \%(6 I)$

clearly stated and justified

The form of economic evaluation

used is stated

The choice of form of economic evaluation is justified in relation to the questions addressed

\section{Data collection}

The primary outcome measure(s) for the economic evaluation are clearly stated

Productivity changes (if included) are reported separately

The relevance of productivity changes to the study question is discussed

Quantities of resources are reported separately from their unit costs

Methods of the estimation of quantities and unit costs are described
"The aim of this study was to evaluate the epidemiology, additional length of stay, incremental costs and outcomes due to hospitalacquired infections, and to estimate the potential impact of infection control on community hospitals and medical centers." ${ }^{26}$

"More recently, an argument has been made to focus on direct costs (primarily consumables), because they are most subject to savings by implementation of effective infection control interventions." ${ }^{37}$

"The main focus of costs calculated in this study was the health care sector." 13

"Economic burden of surgical site infections at a European university hospital." 32

"To quantify the economic and medical burden of SSls in a European university hospital, we conducted a matched casecontrol study nested in a larger prospective observational study." 32

"Data on the predicted number of cases of hospital-acquired infection were combined with data on the estimated prolongation of stay due to hospital-acquired infection. This produced an estimate of the number of bed days attributable to hospital-acquired infection. Valuations of the opportunity cost of the resources used to supply a bed day were applied to derive a monetary estimate of the opportunity cost of hospital-acquired infection." ${ }^{30}$

"Lost productivity costs due to hospital staff members on sick leave totaled $€ 9,264$." 58

"Additional expenses were $€ 18,375$ for increased nursing care (extra staffing of temporary isolation ward)." ${ }^{58}$

"All patients staying more than 24 hours in a 19-bed MICU at Barnes-Jewish Hospital from Jul I, 1997 to Dec 3I, 1999 were eligible. All health care workers and visitors were required to wear gowns and gloves on entry into the rooms of patients colonized or infected with VRE from Jul I, 1997 to Jun 30, 1998 and from Jul I, 1999 to Dec 3I, 1999. All patients were actively screened[...]. A matched cohort study design was used to determine the attributable cost of VRE. Patients without VRE from the same [medical intensive care unit] population were matched to patients with VRE by diagnosis-related group (DRG) code, APACHE score, and age.VRE-attributable length of MICU stay (d): 4.0, 18.9, 35.3 VRE-attributable LOS (d): 8.3, 38.2, 18.9."67

"Overall costs for the vancomycin-resistant Enteroccoci surveillance and infection control program were estimated using the hospital's step-down cost allocation system, which recorded line-item cost data per resource consumed and total cost per hospital admission. MICU costs were estimated from these data by dividing the patient's total hospitalization cost by total days of hospitalization and then multiplying the quotient by the patient's total MICU-days. This data system also provided hospital reimbursement data, type of insurance, case-mix index, and DRG. Variable cost

- gown $\$ 0.75$ each

- gloves $\$ 0.07 /$ pair 
Table 2 (Continued)

Methodologic feature

Studies with this feature

Example of information used in the analysis

present \% (n)

Currency and price data are recorded

$80 \%(49)$

$39 \%(24)$

Details of currency of price adjustments for

inflation or currency conversion are given

Analysis and interpretation of results

Time horizon of costs and benefits is stated

$87 \%(53)$

$95 \%(58)$

Details of statistical tests and confidence

intervals are given for stochastic data

The approach to sensitivity analysis is given

$30 \%(18)$

The choice variables for sensitivity analysis

$30 \%(18)$

is justified

The ranges over which the variables are

varied are stated
- hand hygiene $\$ 0.10 /$ use

- nursing time $\$ 27 /$ hour

- isolation cart set up $\$ 18.00$

- VRE-negative $\$ 12.13$

- VRE-positive \$24.29”67

“Costs are reported in 200 I Euros (I€= US \$0.95)."46

"All costs were adjusted to 1999 dollars using the consumer price index for health care." 44

"In the hospital X, a total of 90 persons with symptoms and signs consistent with norovirus gastroenteritis with clinical onsets in the time period from Dec I, 2006 to Feb 13, 2007 were reported." 58

"Using logistic regression, preoperative antibiotic therapy (cefazolin/metronidazole vs cefotetan), patient demographics, surgical procedure, obesity, and modified SECNIC score were examined as predictors of LOS $\geq 1$ week and cost $\geq \$ 15,000$." 34 "Models 2 and 3 incorporated additional cost predictors sequentially: suspected and confirmed $\mathrm{HAI}$ and ICU treatment." ${ }^{23}$ "Several parameters were changed to determine the impact of our four main assumptions on the net benefits of gowns." ${ }^{\prime 67}$ "The variation between 60 to 140 patient contacts yielded net benefits of $\$ 388,664$ and $\$ 450,017$, respectively. The variable of I to 4 cultures per patients resulted in net benefits of $\$ 418$, 188 and $\$ 421,464$, respectively. The variation in costs of labor and materials results in net benefits of $\$ 406,488$ and $\$ 435,426$,

respectively." 67

Major outcomes are presented in a
"Cost data were available for all of the 164 patients admitted after Jul I, 1999." 45

"In conclusion, in the presence of prompt catheter removal and initiation of antimicrobial therapy, no significant attributable mortality could be documented in critically ill patients. However, increases in the durations of ICU and hospital stay contribute to an important economic burden. These significant increases in cost underscore the need to vigorous application of evidence-based cost-effective preventive measures." ${ }^{38}$

"Prevention of MRSA infection is essential if we are to minimize its major impact on individual patients and if we are to get the most effective use of health care resources." ${ }^{4}$

"It should be understood that the cost of an infection, if avoided, will not be realized as a cash saving. Many of the costs/benefits are fixed and it is principally the variable costs/benefits (for example drugs and other consumables), which represent a small proportion of the total costs, that would show as cash savings, and as such an expenditure that could be avoided." 27

Notes: ${ }^{W}$ When the major outcomes are presented in aggregated form the overall cost is stated, for example, surgical site infections cost $\$ 10,000$. If the major outcomes are presented in disaggregated form the different components that make up the overall cost is stated. For example, surgical site infections cost $\$ 10,000$, of that professional costs were $\$ 3000$, medication costs were $\$ 2000$ and hospitalization costs were $\$ 5000$. (Numbers used are for illustration only).

Abbreviations: LOS, length of stay; MRSA, methicillin-resistant Staphylococcus aureus; VRE, vancomycin-resistant Enterococcus.

cesarean delivery were $\$ 2888$ to $\$ 3574^{37}$ per case. The latter study found similar attributable costs for surgical site infections using two different statistical methods for attributing costs (attributable cost for surgical site infection was $\$ 3529$ by regression and $\$ 2852$ by propensity score). ${ }^{37}$

\section{Nosocomial bloodstream infections}

We found ten studies of the economic burden of nosocomial blood stream infections (one prospective, three retrospective cohorts, five retrospective case controls, and one case series). 
In general European patient populations, nosocomial bloodstream infection was associated with costs ranging from $\$ 2604$ to $\$ 22,414 .^{22,31,38-41}$ One American study reported average incremental costs of $\$ 21,013 .{ }^{42}$ In a pediatric intensive care unit (ICU), nosocomial bloodstream infection was estimated to cost $\$ 49,663 .{ }^{43}$ Very-low-birth-weight infants with nosocomial bloodstream infection incurred average total costs $\$ 71,384$ higher than those without the infection. ${ }^{44}$ S. aureus bacteremia in patients with prosthetic implants was associated with $\$ 81,743$ in costs per nosocomial case in one prospective case series. ${ }^{45}$

\section{Nosocomial sepsis}

We found two studies of the economic burden of nosocomial sepsis. In one retrospective cohort study, nosocomial sepsis was associated with mean additional costs of $\$ 33,872^{46}$ per case. In one prospective cohort, ICU-acquired sepsis was associated with a mean increase of $\$ 44,178$ in total costs per case. ${ }^{47}$

\section{Nosocomial rotavirus infection}

We reviewed three studies of nosocomial rotavirus infection. One prospective cohort study estimated the costs associated with nosocomial rotavirus infection in children under 30 months of age, but did not provide a per-case result; this study estimated that the national cost of all cases in 1 year in Italy is $\$ 11,952,319 .{ }^{48}$ Rotavirus in children under 48 months of age was associated with $\$ 3591$ in costs per case in one prospective case series. ${ }^{49}$ One prospective study with a nested case control reported $\$ 2210$ in mean excess costs per case. ${ }^{50}$

\section{Nosocomial urinary tract infection}

We found four studies of the economic burden of nosocomial urinary tract infections. These included one prospective, two retrospective cohorts, and one retrospective case control study. The average costs attributable to urinary tract infection ranged from $\$ 788$ to $\$ 18,717.22,31,51,52$

\section{Nosocomial pneumonia}

We found four studies of the economic burden of nosocomial pneumonia. Two studies were prospective cohort studies and found that nosocomial pneumonia was associated with average additional costs of $\$ 856^{53}$ and $\$ 23,624 .{ }^{35}$ One German article detailed both a prospective case control and a retrospective case control, reporting average excess costs of $\$ 10,387$ and $\$ 21,057$, respectively. ${ }^{54}$ In one study, the average cost attributable to ventilator-associated pneumonia in a pediatric ICU was $\$ 55,333 .{ }^{55}$

\section{Nosocomial respiratory tract infection}

We included three studies on the economic burden of nosocomial respiratory tract infections: one retrospective cohort, one retrospective case control, and one case control study.

Respiratory tract infections were associated with additional mean costs of $\$ 3476^{31}$ and $\$ 4509^{22}$ in two studies, respectively. In one additional study, a case was defined as an infection of nosocomial respiratory syncytial virus; this infection was associated with mean costs of $\$ 13,083$ per case. ${ }^{56}$

\section{Miscellaneous nosocomial infections}

We included 12 studies that described the economic burden of miscellaneous nosocomial infections. During a Pseudomonas aeruginosa outbreak, it was retrospectively estimated that infected patients who had been on mechanical ventilation incurred excess costs of $\$ 26,522 .{ }^{57}$ Another retrospective case series investigated the economic impact of a norovirus outbreak that affected patients and staff, and did not provide a per-case cost estimate; dividing the total outbreak costs by the given number of case infections yielded a crude estimate of $\$ 1282$ per case; ${ }^{58}$ another similar study yielded an estimate of $\$ 2972$ per case of outbreak-related norovirus. ${ }^{59}$ In one drug-resistant Salmonella typhimurium outbreak in a Turkish neonatal ICU, cases incurred charges \$1208 higher than controls. ${ }^{60} \mathrm{~A}$ pertussis outbreak incurred total hospital costs of $\$ 34,956$ and $\$ 50,668$ in two hospitals. ${ }^{61}$ The attributable costs during a Salmonella outbreak in one Australian tertiary care complex were reported in total costs rather than per case, and dividing by the number of cases yields an estimate of $\$ 2552$ per case. ${ }^{62}$ One retrospective case control study defined a case as a multidrug-resistant infection of Acinetobacter baumannii in burn patients, and reported a mean additional cost per case of $\$ 121,371 .{ }^{63}$ In one Irish hospital, postoperative MRSA infection incurred additional costs of $\$ 13,651 .^{64}$ Another retrospective case series reported the attributable cost of MRSA to be $\$ 123,367$ and vancomycin-resistant Enterococcus (VRE) to be $\$ 128,690 .{ }^{65}$ One prospective study with a nested case control reported a median incremental cost of $\$ 9708$ per case of Clostridium difficile-associated disease (CDAD) ${ }^{66}$ One case control study reported the attributable costs of VRE in the medical ICU and in hospital to be $\$ 9543$ and $\$ 14,532$, respectively. ${ }^{67}$ One retrospective cohort study examined the cost associated with potentially preventable complications and found it to be $\$ 634,432,559 .{ }^{68}$ 
Table 3 Detailed summary of systematic review

\begin{tabular}{|c|c|c|c|c|}
\hline $\begin{array}{l}\text { Study, } \\
\text { methodologic } \\
\text { feature score }\end{array}$ & Design & $\begin{array}{l}\text { Method for } \\
\text { estimating } \\
\text { attributable cost }\end{array}$ & Resources measured & $\begin{array}{l}\text { Source of resource cost } \\
\text { (currency, year) }\end{array}$ \\
\hline \multicolumn{5}{|c|}{ General studies of adverse events and adverse drug events } \\
\hline $\begin{array}{l}\text { Hoonhout }{ }^{13} \\
\text { Methodologic } \\
\text { feature } \\
\text { score }=16\end{array}$ & $\begin{array}{l}\text { Retrospective } \\
\text { cohort }\end{array}$ & $\begin{array}{l}\text { Multivariate } \\
\text { multilevel analysis }\end{array}$ & $\begin{array}{l}\text { Direct medical costs, based on } \\
\text { additional LOS and additional } \\
\text { medical procedures }\end{array}$ & $\begin{array}{l}\text { Dutch guideline prices of 2003, } \\
\text { corrected for } 2004 \text { (€, 2004) }\end{array}$ \\
\hline $\begin{array}{l}\text { Ehsani }{ }^{14} \\
\text { Methodologic } \\
\text { feature } \\
\text { score }=13\end{array}$ & $\begin{array}{l}\text { Retrospective } \\
\text { cohort }\end{array}$ & $\begin{array}{l}\text { Simple linear } \\
\text { regression modeling }\end{array}$ & $\begin{array}{l}\text { Total cost of per-patient care from } \\
\text { database (not further described) }\end{array}$ & $\begin{array}{l}\text { Patient-level costing dataset of the } \\
\text { Victorian Department of } \\
\text { Human Services (AU\$, year unclear) }\end{array}$ \\
\hline $\begin{array}{l}\text { New }^{15} \\
\text { Methodologic } \\
\text { feature } \\
\text { score }=12\end{array}$ & $\begin{array}{l}\text { Retrospective } \\
\text { cohort }\end{array}$ & $\begin{array}{l}\text { Ordinary least squares } \\
\text { regression analysis }\end{array}$ & $\begin{array}{l}\text { LOS, surgical and medical procedures, } \\
\text { laboratory tests }\end{array}$ & $\begin{array}{l}\text { Hospital accounting database } \\
\text { (AU\$, 2004) }\end{array}$ \\
\hline $\begin{array}{l}\text { Pappas }^{16} \\
\text { Methodologic } \\
\text { feature } \\
\text { score }=12\end{array}$ & $\begin{array}{l}\text { Retrospective } \\
\text { cohort }\end{array}$ & Regression analysis & $\begin{array}{l}\text { Nursing staff hours per patient day, } \\
\text { clinical outcomes, patient-level data }\end{array}$ & $\begin{array}{l}\text { Cost accounting system/ } \\
\text { I Eclipsys TSI (US\$, year unstated) }\end{array}$ \\
\hline $\begin{array}{l}\text { Morris }{ }^{17} \\
\text { Methodologic } \\
\text { feature } \\
\text { score }=12\end{array}$ & $\begin{array}{l}\text { Retrospective } \\
\text { cohort }\end{array}$ & Unclear & $\begin{array}{l}\text { Hospital charges, costs, legal fees and } \\
\text { indemnity payments, legal write-offs }\end{array}$ & $\begin{array}{l}\text { Unclear (assumed US\$, } \\
\text { year unstated) }\end{array}$ \\
\hline $\begin{array}{l}\text { Aoki }{ }^{18} \\
\text { Methodologic } \\
\text { feature } \\
\text { score }=15\end{array}$ & Case series & $\begin{array}{l}\text { Multivariate logistic } \\
\text { analysis }\end{array}$ & Legal compensation in medical disputes & $\begin{array}{l}\text { Medical dispute records } \\
\text { (US\$, 2007; converted from JP yen) }\end{array}$ \\
\hline $\begin{array}{l}\text { Kaushal }^{19} \\
\text { Methodologic } \\
\text { feature } \\
\text { score }=14\end{array}$ & $\begin{array}{l}\text { Prospective with } \\
\text { nested } \\
\text { case control }\end{array}$ & $\begin{array}{l}\text { Matched case-control, } \\
\text { linear regression model }\end{array}$ & $\begin{array}{l}\text { Charges, actual variable costs, } \\
\text { actual fixed costs, actual direct variable } \\
\text { costs and actual direct fixed costs }\end{array}$ & $\begin{array}{l}\text { Hospital TSI database } \\
\text { (US\$, 2003) }\end{array}$ \\
\hline $\begin{array}{l}\text { Senst }{ }^{20} \\
\text { Methodologic } \\
\text { feature } \\
\text { score }=13\end{array}$ & $\begin{array}{l}\text { Prospective with } \\
\text { nested } \\
\text { case control }\end{array}$ & $\begin{array}{l}\text { Case control, } \\
\text { multiple linear } \\
\text { regression model }\end{array}$ & Charges converted to costs & $\begin{array}{l}\text { Prospectively recorded charges } \\
\text { (US\$, year unclear) }\end{array}$ \\
\hline Nosocomial inf & ections (not other & ise specified) $)^{a}$ & & \\
\hline $\begin{array}{l}\text { Chen }^{21} \\
\text { Methodologic } \\
\text { feature } \\
\text { score }=15\end{array}$ & $\begin{array}{l}\text { Retrospective } \\
\text { analytic cohort }\end{array}$ & $\begin{array}{l}\text { Stratified analysis and } \\
\text { regression model }\end{array}$ & $\begin{array}{l}\text { LOS, physician services, medical and } \\
\text { surgical procedures, lab and radiology, } \\
\text { unit costs }\end{array}$ & $\begin{array}{l}\text { Hospital database } \\
\text { (US\$, 200I) }\end{array}$ \\
\hline $\begin{array}{l}\text { Chen }^{22} \\
\text { Methodologic } \\
\text { feature } \\
\text { score }=15\end{array}$ & $\begin{array}{l}\text { Retrospective } \\
\text { analysis of a } \\
\text { prospectively } \\
\text { assembled cohort }\end{array}$ & $\begin{array}{l}\text { Generalized linear } \\
\text { modeling }\end{array}$ & $\begin{array}{l}\text { Medical and surgical procedures, } \\
\text { medications, lab investigation, } \\
\text { ICU bed-days }\end{array}$ & $\begin{array}{l}\text { Hospital database, microcosting } \\
\text { (US\$, 2007, converted from } \\
\text { Taiwanese dollars) }\end{array}$ \\
\hline $\begin{array}{l}\text { Roberts }^{23} \\
\text { Methodologic } \\
\text { feature } \\
\text { score }=16\end{array}$ & $\begin{array}{l}\text { Retrospective } \\
\text { cohort }\end{array}$ & $\begin{array}{l}\text { Ordinary least-squares } \\
\text { regression and } \\
\text { economic models }\end{array}$ & $\begin{array}{l}\text { Location of care (ICU, ward, etc), } \\
\text { lab and radiography tests, procedures, } \\
\text { consultations and medication }\end{array}$ & $\begin{array}{l}\text { Data abstracted from medical } \\
\text { records, microcosting (US\$, 1998) }\end{array}$ \\
\hline
\end{tabular}




\begin{tabular}{|c|c|c|c|c|}
\hline $\begin{array}{l}\text { Sample population } \\
\text { (time horizon) }\end{array}$ & Case definition & Incidence & $\begin{array}{l}\text { Attributable } \\
\text { LOS }\end{array}$ & $\begin{array}{l}\text { Estimated } \\
\text { attributable cost }\end{array}$ \\
\hline $\begin{array}{l}7926 \text { patients of which } \\
45 \text { I patients with AEs in } \\
\text { 2I Dutch hospitals } \\
\text { (Aug 2005-Oct 2006) }\end{array}$ & $\begin{array}{l}\text { Any } A E \text { : an unintended injury } \\
\text { resulting in temporary/ } \\
\text { permanent disability, death, } \\
\text { extra LOS, caused by health care }\end{array}$ & $5.7 \%$ & $\begin{array}{l}\text { University hospitals: } \\
\text { I0.I additional days } \\
\text { General: } \\
8.9 \text { additional days }\end{array}$ & $\begin{array}{l}\text { Attributable costs of all } \\
\text { AEs: mean } € 4446 \text { per } A E \\
\text { Excess costs of } \\
\text { preventable } A E s \text { : mean } \\
€ 3634 \text { per } A E\end{array}$ \\
\hline $\begin{array}{l}\text { Of } 979,834 \text { admissions, } \\
67,609 \text { had an AE, } \\
45 \text { hospitals in Victoria, Australia } \\
\text { (Jun 2003- } \\
\text { Jul 2004) }\end{array}$ & $\begin{array}{l}\text { Any } A E \text {, identified via diagnosis } \\
\text { codes }\end{array}$ & $6.9 \%$ had at least I AE & 10 additional days & $\mathrm{AU} \$ 1 \mathrm{I}, 846$ per $\mathrm{AE}$ \\
\hline $\begin{array}{l}\text { Of } 1605 \mathrm{SCl} \\
\text { patients, } 610 \text { with one } \\
\text { complication, } \\
\text { in } 45 \text { campuses of } \\
26 \mathrm{AU} \text { health services } \\
\text { (Jun 2003-Jun 2004) }\end{array}$ & $\begin{array}{l}\text { At least one } \mathrm{AE} \text { or } \mathrm{HAC} \\
\text { in a patient with } \mathrm{SCl}\end{array}$ & $\begin{array}{l}38 \% \text { of multiday } \mathrm{SCl} \\
\text { episodes had at least } \\
\mathrm{I} \text { incident complication }\end{array}$ & 32 additional days & $\begin{array}{l}\text { Additional costs, any } \\
\text { complication: AU } \$ 7359 \\
\text { UTI: AU } \$ 23,705 \\
\text { Procedural complications: } \\
\text { AU } \$ 2 \text { I,82। } \\
\text { Anemia: AU } \$ 18,047 \\
\text { Pressure ulcer: AU } \$ 17,882\end{array}$ \\
\hline $\begin{array}{l}\text { Of } 3200 \text { inpatients. } \\
\text { Medical patients: } \\
688 \text { Surgical patients: } 461 ; 2 \\
\text { hospitals from hospital databases } \\
\text { ( } 24 \text { month) }\end{array}$ & $\begin{array}{l}\text { Nosocomial AEs including } \\
\text { medication error, fall, UTI, } \\
\text { pneumonia, and pressure ulcer }\end{array}$ & $\begin{array}{l}\text { Medical patients: } \\
21.5 \% \text { surgical } \\
\text { patients: } 14.4 \%\end{array}$ & Not available & $\begin{array}{l}\text { Medical patients: } \\
\$ 1029 \text { per } A E \\
\text { Surgical patients: } \\
\$ 903 \text { per } A E\end{array}$ \\
\hline $\begin{array}{l}\text { I } 30 \text { cases out of } 32,100 \text { patients } \\
\text { over age I3 } \\
\text { (Jan I, 1995-Dec 6, 1999) }\end{array}$ & $\begin{array}{l}\text { Surgical AEs, not further } \\
\text { specified }\end{array}$ & $0.4 \%$ & Not available & $\begin{array}{l}\text { Total legal payment for } \\
\text { the study group ( } 126) \\
\text { was } \$ 8.2 \text { million }\end{array}$ \\
\hline $\begin{array}{l}\text { I55 resolved medical dispute } \\
\text { cases in Japan (1989-1998) }\end{array}$ & $\begin{array}{l}\text { Any medical dispute case } \\
\text { resolved during the study } \\
\text { period }\end{array}$ & Not available & Not available & $\begin{array}{l}\text { Legal compensation for } \\
\text { an } A E \text { claim was mean } \\
\$ 38,937 \text {, median } \$ 7417\end{array}$ \\
\hline $\begin{array}{l}\text { I08 cases matched with } \\
375 \text { controls in I hospital MICU } \\
\text { and cardiac care unit } \\
\text { (Jul 2002-Jun 2003) }\end{array}$ & $\begin{array}{l}\text { Any AE, detected via } \\
\text { observation, reports, } \\
\text { and guided implicit chart } \\
\text { abstraction }\end{array}$ & Not available & $\begin{array}{l}\text { MICU, AEs: } \\
0.77 \text { additional days } \\
\text { Cardiac care unit AEs: } \\
\text { I.08 additional days }\end{array}$ & $\begin{array}{l}\$ 396 I \text { in the MICU, } \\
\$ 3857 \text { in the cardiac care } \\
\text { unit }\end{array}$ \\
\hline $\begin{array}{l}\text { Of } 3187 \text { admissions I } 34 \text { had } \\
\text { an ADE, in I US health care } \\
\text { network including } 4 \text { hospitals } \\
\text { and } 26 \text { clinics (53-day study } \\
\text { period, 1998) }\end{array}$ & $\begin{array}{l}\text { ADE: an injury caused by the } \\
\text { use, disuse or misuse of a drug } \\
\text { via error or despite proper } \\
\text { usage }\end{array}$ & $4.2 \%$ & 1.2 additional days & $\$ 2162$ per ADE \\
\hline $\begin{array}{l}778 \text { patients admitted to } \\
3 \text { ICUs in I hospital between } \\
\text { Oct } 200 \text { I and Jun } 2002\end{array}$ & $\begin{array}{l}\text { Any nosocomial infection } \\
\text { (such as BSI, UTI, SSI) } \\
\text { confirmed by culture, } \\
\text { symptoms and an attending } \\
\text { physician }\end{array}$ & $\begin{array}{l}\text { I0.2\% had at } \\
\text { least one } \\
\text { nosocomial } \\
\text { infection }\end{array}$ & I8.2 additional days & $\begin{array}{l}\$ 3306 \text { additional costs } \\
\text { per nosocomial infection }\end{array}$ \\
\hline $\begin{array}{l}40 \mathrm{I} \mathrm{Nls} \mathrm{in} 320 \text { of } 2757 \text { patients, } \\
\text { in } 4 \text { ICUs in one hospital in } \\
\text { Taiwan } \\
(2003-2004)\end{array}$ & $\begin{array}{l}\text { BSI, UTI, SSI, respiratory tract } \\
\text { infection "and others" } \\
\text { diagnosed } \geq 48 \text { hours after } \\
\text { admission to ICU }\end{array}$ & $\begin{array}{l}\text { 14.5 } \mathrm{NI} \text { episodes } \\
\text { per } 100 \text { admissions }\end{array}$ & Not available & $\begin{array}{l}\$ 10,015 \text { attributable } \\
\text { cost per case }\end{array}$ \\
\hline $\begin{array}{l}25 \text { patients with } \mathrm{HAl} \text { in one } \\
\text { urban teaching hospital } \\
\text { (Jan-Dec 1998) }\end{array}$ & $\begin{array}{l}\text { Any HAls, according to the } \\
\text { CDC's NNIS }\end{array}$ & $15.2 \%$ & I 0.7 additional days & $\begin{array}{l}\text { Incremental costs } \\
\text { attributable to suspected } \\
\text { HAl: } \$ 6767 \\
\text { confirmed HAI: } \$ 15,275\end{array}$ \\
\hline
\end{tabular}


Table 3 (Continued)

\begin{tabular}{|c|c|c|c|c|}
\hline $\begin{array}{l}\text { Study, } \\
\text { methodologic } \\
\text { feature score }\end{array}$ & Design & $\begin{array}{l}\text { Method for } \\
\text { estimating } \\
\text { attributable cost }\end{array}$ & Resources measured & $\begin{array}{l}\text { Source of resource cost } \\
\text { (currency, year) }\end{array}$ \\
\hline $\begin{array}{l}\text { Kilgore }^{24} \\
\text { Methodologic } \\
\text { feature } \\
\text { score }=13\end{array}$ & $\begin{array}{l}\text { Retrospective } \\
\text { cohort }\end{array}$ & $\begin{array}{l}\text { Multivariable } \\
\text { regression models } \\
\text { and restricted models }\end{array}$ & $\begin{array}{l}\text { Total, variable costs of inpatient care, } \\
\text { LOS }\end{array}$ & $\begin{array}{l}\text { Cardinal Health-MedMined } \\
\text { database (US\$, 2007) }\end{array}$ \\
\hline $\begin{array}{l}\text { Esatoglu }{ }^{25} \\
\text { Methodologic } \\
\text { feature } \\
\text { score }=12\end{array}$ & $\begin{array}{l}\text { Retrospective } \\
\text { case control }\end{array}$ & $\begin{array}{l}\text { Matched I:I by } \\
\text { age, sex, clinic, } \\
\text { primary diagnosis } \\
\text { of the infected patients }\end{array}$ & $\begin{array}{l}\text { LOS, medical goods/materials, drugs, } \\
\text { tests, beds, treatments and other costs. }\end{array}$ & $\begin{array}{l}\text { Unspecified, presumably hospital } \\
\text { accounting database (US\$, 200I) }\end{array}$ \\
\hline $\begin{array}{l}\text { Sheng } \\
\text { Methodologic } \\
\text { feature } \\
\text { score }=12\end{array}$ & $\begin{array}{l}\text { Retrospective } \\
\text { case control }\end{array}$ & $\begin{array}{l}\text { Matched I:I by } \\
\text { age } \pm 2 \text {, sex, } \\
\text { underlying illness, } \\
\text { operation(s), } \\
\text { admission date } \\
28 \text { days, ward, } \\
\text { diagnosis and severity }\end{array}$ & $\begin{array}{l}\text { Costs of stay, medication, } \\
\text { lab procedures, materials } \\
\text { and services, nursing care }\end{array}$ & $\begin{array}{l}\text { Hospital finance departments } \\
\text { (US\$, 2002) }\end{array}$ \\
\hline $\begin{array}{l}\text { Plowman }^{27} \\
\text { Methodologic } \\
\text { feature } \\
\text { score }=16\end{array}$ & $\begin{array}{l}\text { Prospective } \\
\text { cohort }\end{array}$ & $\begin{array}{l}\text { Linear regression } \\
\text { model }\end{array}$ & $\begin{array}{l}\text { Resources, LOS, care and treatment, } \\
\text { paid staff time, nursing costs, } \\
\text { unit costs for lab, radiology and } \\
\text { other diagnostic procedures }\end{array}$ & $\begin{array}{l}\text { Costs estimated for specialty } \\
\text { via interviewing health care } \\
\text { professionals, hospital database } \\
\text { (GBP, year unclear) }\end{array}$ \\
\hline $\begin{array}{l}\mathrm{Lee}^{28} \\
\text { Methodologic } \\
\text { feature } \\
\text { score }=13\end{array}$ & $\begin{array}{l}\text { Retrospective } \\
\text { cohort }\end{array}$ & $\begin{array}{l}\text { Linear regression } \\
\text { models }\end{array}$ & $\begin{array}{l}\text { Third part payer's overall } \\
\text { hospital costs, increased } \\
\text { LOS (postsurgical), antibiotic costs }\end{array}$ & $\begin{array}{l}\text { Quality Indicator/Improvement } \\
\text { Project database (US\$, 2007, } \\
\text { converted from JP yen) }\end{array}$ \\
\hline $\begin{array}{l}\text { Mahieu }^{29} \\
\text { Methodologic } \\
\text { feature } \\
\text { score = I5 }\end{array}$ & $\begin{array}{l}\text { Retrospective } \\
\text { cohort with } \\
\text { nested } \\
\text { case control }\end{array}$ & $\begin{array}{l}\text { Matched by gestational } \\
\text { age and early post-natal } \\
\text { co-morbidity factors }\end{array}$ & Charges and LOS & $\begin{array}{l}\text { Charges from hospital discharge } \\
\text { abstracts and patient files (€, 1995) }\end{array}$ \\
\hline $\begin{array}{l}\text { Graves }^{30} \\
\text { Methodologic } \\
\text { feature } \\
\text { score = I5 }\end{array}$ & Decision model & Monte Carlo simulation & $\begin{array}{l}\text { Estimated literature cost per bed-day, } \\
\text { literature estimates of increased LOS, } \\
\text { medical and surgical services }\end{array}$ & $\begin{array}{l}\text { Database and literature values } \\
\text { for NZ hospitals (US\$, year unstated) }\end{array}$ \\
\hline $\begin{array}{l}\text { Chen }^{22} \\
\text { Methodologic } \\
\text { feature } \\
\text { score }=15\end{array}$ & $\begin{array}{l}\text { Retrospective } \\
\text { analysis of a } \\
\text { prospectively } \\
\text { assembled cohort }\end{array}$ & $\begin{array}{l}\text { Generalized linear } \\
\text { modeling }\end{array}$ & $\begin{array}{l}\text { Medical and surgical procedures, } \\
\text { medications, lab investigation, } \\
\text { ICU bed-days }\end{array}$ & $\begin{array}{l}\text { Hospital database, microcosting } \\
\text { (US\$, 2007, converted from } \\
\text { Taiwanese dollars) }\end{array}$ \\
\hline $\begin{array}{l}\text { Defez }{ }^{31} \\
\text { Methodologic } \\
\text { feature } \\
\text { score }=15\end{array}$ & $\begin{array}{l}\text { Retrospective } \\
\text { case control }\end{array}$ & $\begin{array}{l}\text { Matched I:I by age, } \\
\text { sex, ward, LOS before } \\
\text { infection, DRG, and } \\
\text { McCabe index }\end{array}$ & $\begin{array}{l}\text { Lab tests, radiology, surgery, } \\
\text { antimicrobial agents, } \\
\text { rate per day of hospital bed }\end{array}$ & $\begin{array}{l}\text { Reimbursement from La Nomeclature } \\
\text { Générale des Actes Professionnels and } \\
\text { hospital pharmacy accounting } \\
\text { database (€, 2004) }\end{array}$ \\
\hline $\begin{array}{l}\text { Weber }{ }^{32} \\
\text { Methodologic } \\
\text { feature } \\
\text { score }=17\end{array}$ & $\begin{array}{l}\text { Prospective with } \\
\text { nested } \\
\text { case control }\end{array}$ & $\begin{array}{l}\text { Matched I:I by } \\
\text { age } \pm 5 \text { years, } \\
\text { procedure code, } \\
\text { and NNIS risk index }\end{array}$ & $\begin{array}{l}\text { LOS, ICU LOS, patient charges, } \\
\text { antibiotic costs }\end{array}$ & $\begin{array}{l}\text { Microcosting from hospital } \\
\text { accounting database } \\
\text { (Swiss franc, assumed 200I) }\end{array}$ \\
\hline
\end{tabular}




\begin{tabular}{|c|c|c|c|c|}
\hline $\begin{array}{l}\text { Sample population } \\
\text { (time horizon) }\end{array}$ & Case definition & Incidence & $\begin{array}{l}\text { Attributable } \\
\text { LOS }\end{array}$ & $\begin{array}{l}\text { Estimated } \\
\text { attributable cost }\end{array}$ \\
\hline $\begin{array}{l}\text { Of I,355,647 admissions, } \\
58,293 \text { had an NIM. } \\
\text { Over } 69 \text { months from } \\
55 \text { hospital databases } \\
\text { (Mar 200I-Jan 2006) }\end{array}$ & $\begin{array}{l}\text { Any nosocomial infection, } \\
\text { identified via NIM }\end{array}$ & $\begin{array}{l}\text { Overall NIM rate } \\
\text { was } 4.3 \%\end{array}$ & 5.4 additional days & $\begin{array}{l}\text { NIMs are associated } \\
\text { with attributable costs } \\
\text { of US } \$ 12,197\end{array}$ \\
\hline $\begin{array}{l}57 \text { patients with HAI } \\
\text { matched I:I, in one hospital } \\
\text { in Ankara, Turkey } \\
\text { (Sep-Dec 200I) }\end{array}$ & $\begin{array}{l}\text { Any HAl, not further } \\
\text { described }\end{array}$ & Not available & Mean 23 additional days & $\begin{array}{l}\text { HAI mean additional cost: } \\
\text { US } \$ 2026.70\end{array}$ \\
\hline $\begin{array}{l}273 \text { adult case-control pairs, } \\
\text { from } 2 \text { community hospitals } \\
\text { and I tertiary medical } \\
\text { center (Oct-Dec 2002) }\end{array}$ & $\begin{array}{l}\text { Patients aged } \geq 16 \text { years with } \\
\text { onset of any infection } \geq 48 \text { hours } \\
\text { after admission or within I week } \\
\text { of discharge }\end{array}$ & Not available & I9.67 additional days & $\begin{array}{l}\text { US\$5I89 in mean } \\
\text { additional costs }\end{array}$ \\
\hline $\begin{array}{l}4000 \text { adults in one general } \\
\text { hospital in London, } \\
\text { England, of which } 312 \text { had } \\
\text { an HAI (Apr 1994- } \\
\text { May 1995) }\end{array}$ & Any HAI & $\begin{array}{l}\text { Incidence of } \\
\text { HAls: } 7.8 \%\end{array}$ & I4.I additional days & $\begin{array}{l}\text { Mean additional costs due } \\
\text { to } \mathrm{HAl} \text { at any site: } £ 3 \mid 54 \\
\text { (model estimate } £ 29 \mid 7 \text { ) }\end{array}$ \\
\hline $\begin{array}{l}\text { I058 gastrectomy } \\
\text { patients from } \\
\text { I0 JP hospitals, } \\
\text { of which } 215 \text { had any HAI } \\
\text { (Apr 2004-Jan 2007) }\end{array}$ & Diagnosed with any HAI & $\begin{array}{l}\text { HAl incidence } \\
20.3 \%\end{array}$ & I0.6 days attributable & $\begin{array}{l}\text { Attributable HAI costs: } \\
\text { US\$2767 } \\
\text { (range } \$ 1035-\$ 6513 \text { ) }\end{array}$ \\
\hline $\begin{array}{l}\text { Of } 515 \text { neonates in one } \\
\text { Belgian NICU, } 69 \text { had } \\
\text { one or more HAI } \\
\text { (Oct 1993-Dec 1995) }\end{array}$ & $\begin{array}{l}\text { Infections } \geq 48 \text { hours after } \\
\text { admission to NICU and } \\
\text { treated with IV antibiotics } \\
\text { for } 5+\text { days were considered } \\
\text { nosocomial }\end{array}$ & $\begin{array}{l}13 \% \text { incidence of } \\
\text { one or more } \mathrm{HAI}\end{array}$ & Mean 24 additional days & $\begin{array}{l}\text { Mean extra charge with } \\
\text { HAI was } € I I, 750\end{array}$ \\
\hline $\begin{array}{l}\text { Any/all recorded admissions, NZ } \\
\text { hospitals (1998-1999) }\end{array}$ & HAl reported in database & $\begin{array}{l}\text { No overall incidence } \\
\text { reported }\end{array}$ & Not collected in study & $\begin{array}{l}\text { Not reported per case. } \\
\text { Estimated national costs } \\
\text { of HAl over fiscal year } \\
\text { in NZ, medical patients: } \\
\mathrm{NZ} \$ 4,569,826 \text {. } \\
\text { Surgical: NZ } \$ 3,900,922\end{array}$ \\
\hline $\begin{array}{l}40 \text { I NIs in } 320 \text { of } 2,757 \text { patients, } \\
\text { in } 4 \text { ICUs in one hospital in } \\
\text { Taiwan } \\
(2003-2004)\end{array}$ & $\begin{array}{l}\text { BSI, UTI, SSI, respiratory tract } \\
\text { infection "and others" } \\
\text { diagnosed } \geq 48 \text { hours after } \\
\text { admission to ICU }\end{array}$ & $\begin{array}{l}\text { I4.5 } \mathrm{NI} \text { episodes } \\
\text { per } 100 \text { admissions }\end{array}$ & Not available & $\begin{array}{l}\text { US } \$ 10,0 I 5 \text { attributable } \\
\text { cost per case }\end{array}$ \\
\hline $\begin{array}{l}\text { I } 703 \text { infected patients } \\
\text { from previous study; } \\
30 \text { randomly chosen for } \\
\text { each infection site, total I50. } \\
\text { One French hospital. } \\
(200 \text { I-2003) }\end{array}$ & $\begin{array}{l}\text { Patients with single-site } \\
\text { nosocomial infection }\end{array}$ & Not available & Not available & $\begin{array}{l}\text { Attributable cost } \\
\text { (mean } € \text { ) by site } \\
\text { of infection, } \\
\text { UTI: } € 574 \\
\text { Surgical site: } €|8| 4 \\
\text { Respiratory tract: } € 242 \mid \\
\text { Bloodstream: } € 953 \\
\text { Other: } € \mid 259\end{array}$ \\
\hline $\begin{array}{l}6283 \text { surgical procedures in } \\
\text { one Swiss hospital, } \\
\text { I } 87 \text { with SSI (2000-200I) }\end{array}$ & $\begin{array}{l}\text { All surgical site infections at } \\
\text { one Swiss hospital }\end{array}$ & $3.0 \%$ & I6.8 additional days & $\begin{array}{l}\text { Mean additional hospital } \\
\text { cost was } 19,638 \\
\text { Swiss francs }\end{array}$ \\
\hline
\end{tabular}


Table 3 (Continued)

\begin{tabular}{|c|c|c|c|c|}
\hline $\begin{array}{l}\text { Study, } \\
\text { methodologic } \\
\text { feature score }\end{array}$ & Design & $\begin{array}{l}\text { Method for } \\
\text { estimating } \\
\text { attributable cost }\end{array}$ & Resources measured & $\begin{array}{l}\text { Source of resource cost } \\
\text { (currency, year) }\end{array}$ \\
\hline $\begin{array}{l}\text { Whitehouse }{ }^{33} \\
\text { Methodologic } \\
\text { feature } \\
\text { score }=13\end{array}$ & $\begin{array}{l}\text { Prospective } \\
\text { case control }\end{array}$ & $\begin{array}{l}\text { Matched I:I by } \\
\text { type of operative } \\
\text { procedure, } \\
\text { NNIS risk index, } \\
\text { age } \pm 5 \text {, surgery within } \\
\text { the same year, surgeon }\end{array}$ & $\begin{array}{l}\text { Total direct costs from database, } \\
\text { representing sum of costs required } \\
\text { to provide health care services }\end{array}$ & $\begin{array}{l}\text { Hospital accounting database, } \\
\text { microcosting (US\$, 1997) }\end{array}$ \\
\hline $\begin{array}{l}\text { Mahmoud }{ }^{34} \\
\text { Methodologic } \\
\text { feature } \\
\text { score }=12\end{array}$ & $\begin{array}{l}\text { Retrospective } \\
\text { analytic cohort }\end{array}$ & Logistic regression & $\begin{array}{l}\text { Medical and surgical procedures, } \\
\text { hotel costs, nursing, pharmacy, } \\
\text { ICU, supplies, lab procedures }\end{array}$ & $\begin{array}{l}\text { Large US hospital database: } \\
\text { Premier Perspective database } \\
\text { (US\$, 2005/6) }\end{array}$ \\
\hline $\begin{array}{l}\text { Penel }^{35} \\
\text { Methodologic } \\
\text { feature } \\
\text { score = } 15\end{array}$ & $\begin{array}{l}\text { Prospective } \\
\text { cohort } \\
\text { with a post } \\
\text { hoc analysis }\end{array}$ & Unclear & $\begin{array}{l}\text { LOS. Estimation of per diem cost, } \\
\text { including rooming, lab, medications } \\
\text { and procedure costs }\end{array}$ & $\begin{array}{l}\text { Macro costing: LOS multiplied by } \\
\text { estimation of per diem cost ( } €, 2005)\end{array}$ \\
\hline $\begin{array}{l}\text { Jenney }{ }^{36} \\
\text { Methodologic } \\
\text { feature } \\
\text { score = II }\end{array}$ & $\begin{array}{l}\text { Retrospective } \\
\text { cohort with } \\
\text { nested } \\
\text { case control }\end{array}$ & $\begin{array}{l}\text { Matched I:I by } \\
\text { sex, age } \pm 5 \text {, } \\
\text { NNIS risk index } \\
\text { scores }\end{array}$ & $\begin{array}{l}\text { LOS, antibiotic costs, salaries, } \\
\text { utilities and overhead costs }\end{array}$ & $\begin{array}{l}\text { Hospital finance department } \\
\text { (AU\$, 1999) }\end{array}$ \\
\hline $\begin{array}{l}\text { Olsen }^{37} \\
\text { Methodologic } \\
\text { feature } \\
\text { score = } 12\end{array}$ & $\begin{array}{l}\text { Retrospective } \\
\text { cohort }\end{array}$ & $\begin{array}{l}\text { Generalized least } \\
\text { squares and } \\
\text { propensity score } \\
\text { matched-pairs }\end{array}$ & $\begin{array}{l}\text { Department actual cost components } \\
\text { multiplied by patient charge codes } \\
\text { (pharmacy, room and board, } \\
\text { procedures) }\end{array}$ & $\begin{array}{l}\text { Barnes-Jewish Hospital cost } \\
\text { accounting database (US\$, 2008) }\end{array}$ \\
\hline $\begin{array}{l}\text { Chen }^{22} \\
\text { Methodologic } \\
\text { feature } \\
\text { score }=15\end{array}$ & $\begin{array}{l}\text { Retrospective } \\
\text { analysis of a } \\
\text { prospectively } \\
\text { assembled cohort }\end{array}$ & $\begin{array}{l}\text { Generalized linear } \\
\text { modeling }\end{array}$ & $\begin{array}{l}\text { Medical and surgical procedures, } \\
\text { medications, lab investigation, } \\
\text { ICU bed-days }\end{array}$ & $\begin{array}{l}\text { Hospital database, microcosting } \\
\text { (US\$, 2007, converted from } \\
\text { Taiwanese dollars) }\end{array}$ \\
\hline $\begin{array}{l}\text { Defez } \\
\text { Methodologic } \\
\text { feature } \\
\text { score }=15\end{array}$ & $\begin{array}{l}\text { Retrospective } \\
\text { case control }\end{array}$ & $\begin{array}{l}\text { Matched I:I by age, } \\
\text { sex, ward, LOS before } \\
\text { infection, DRG, and } \\
\text { McCabe index }\end{array}$ & $\begin{array}{l}\text { Lab tests, radiology, surgery, } \\
\text { antimicrobial agents, rate per day } \\
\text { of hospital bed }\end{array}$ & $\begin{array}{l}\text { Reimbursement from La Nomeclature } \\
\text { Générale des Actes Professionnels and } \\
\text { hospital pharmacy accounting } \\
\text { database }(€, 2004)\end{array}$ \\
\hline $\begin{array}{l}\text { Blot }^{38} \\
\text { Methodologic } \\
\text { feature } \\
\text { score }=18\end{array}$ & $\begin{array}{l}\text { Retrospective } \\
\text { case control }\end{array}$ & $\begin{array}{l}\text { Linear regression } \\
\text { analysis, and matched } \\
\text { I:I or I:2 by } \\
\text { APACHE II score, } \\
\text { principal diagnosis, } \\
\text { ICU LOS }\end{array}$ & $\begin{array}{l}\text { Duration of mechanical ventilation, } \\
\text { LOS, hospital costs }\end{array}$ & Patient hospital invoices $(€, 2002)$ \\
\hline $\begin{array}{l}\text { Orsi }^{39} \\
\text { Methodologic } \\
\text { feature } \\
\text { score }=15\end{array}$ & $\begin{array}{l}\text { Retrospective } \\
\text { case control }\end{array}$ & $\begin{array}{l}\text { Matched I: } 2 \text { by } \\
\text { pre-infection } \\
\text { LOS, primary } \\
\text { diagnosis, ward, } \\
\text { CVC, age } \pm 5 \text {, sex }\end{array}$ & Single-day hospital cost, increased LOS & $\begin{array}{l}\text { Data from clinical and micro-biological } \\
\text { records collected by infection control } \\
\text { team ( } € \text {, year unclear) }\end{array}$ \\
\hline
\end{tabular}




\begin{tabular}{|c|c|c|c|c|}
\hline $\begin{array}{l}\text { Sample population } \\
\text { (time horizon) }\end{array}$ & Case definition & Incidence & $\begin{array}{l}\text { Attributable } \\
\text { LOS }\end{array}$ & $\begin{array}{l}\text { Estimated } \\
\text { attributable cost }\end{array}$ \\
\hline $\begin{array}{l}59 \text { cases, each matched } \\
\text { with I control, in one US } \\
\text { hospital (1997-1998) }\end{array}$ & $\begin{array}{l}\text { Orthopedic SSI: superficial } \\
\text { incisional, deep incisional, or } \\
\text { organ/space }\end{array}$ & $\begin{array}{l}\text { Literature rates of } \\
\text { SSI following orthopedic } \\
\text { surgery: } 0.7 \% \text { (low-risk, } \\
\text { hip replacement) to } \\
7.9 \% \text { (high-risk, } \\
\text { spinal fusion) }\end{array}$ & $\begin{array}{l}\text { Median I extra day } \\
\text { during initial hospital stay, } \\
\text { median of } 14 \text { extra days } \\
\text { during follow-up period }\end{array}$ & $\begin{array}{l}\text { Median direct cost was } \\
\text { US } \$ 24,344 \text { for a case, } \\
\text { compared with US } \$ 6636 \\
\text { for uninfected patients }\end{array}$ \\
\hline $\begin{array}{l}25,825 \text { patients undergoing } \\
\text { colorectal procedures, of which } \\
956 \text { have SSI, in US database of } \\
\text { I } 96 \text { hospitals } \\
\text { (Jan 2005-Jun 2006) }\end{array}$ & $\begin{array}{l}\text { Incisional SSIs, superficial } \\
\text { or deep as defined by the } \\
\text { US CDC }\end{array}$ & SSI incidence: $3.7 \%$ & $\begin{array}{l}\text { LOS with postoperative } \\
\text { complications is } 3-\text { II days } \\
\text { longer than without }\end{array}$ & $\begin{array}{l}\text { Mean total direct costs } \\
\text { incurred by treating } \\
\text { SSI: US } \$ 13,746\end{array}$ \\
\hline $\begin{array}{l}26 \mathrm{I} \text { head/neck cancer } \\
\text { surgery patients in one } \\
\text { hospital } \\
\text { SSI: } 94\end{array}$ & $\begin{array}{l}\text { Based on the CDC 1992; } \\
\text { SSI, PP }\end{array}$ & $\begin{array}{l}\text { SSI: } 36 \% \\
\text { PP: } 13 \% \\
\text { SSI and } \\
\text { PP: } 5 \%\end{array}$ & $\begin{array}{l}\text { SSI: } 16 \text { days in } \\
\text { additional mean LOS } \\
\text { PP: I } 7 \text { days } \\
\text { SSI and PP: } 3 \text { I days }\end{array}$ & $\begin{array}{l}\text { SSI: } € 16,000 \text { increase in } \\
\text { mean direct medical costs } \\
\text { PP: } € I 7,000 \\
\text { Both SSI and PP: } € 35,000\end{array}$ \\
\hline
\end{tabular}

PP: 34

SSI and PP: 13

(Jan 1997-Dec 1999)

1377 CABG procedures,

of which 956 had a SSI;

125 cases in an AU

hospital (1996-1998)

1616 women who under-

went low transverse

Patients diagnosed with SSI and/or EM after surgery

SSI after CABG, defined

according to the CDC

SSI incidence: $9.1 \%$

I.36 mean additional days

Incidence of SSI: 5.0\% EM: $7.6 \%$

cesarean delivery at one tertiary

care hospital,

SSI: 8 I

EM: 123

(Jul 1999-Jun 200I)

40I NIs in 320 of 2757 patients, in 4 ICUs in one hospital in

Taiwan

(2003-2004)

1703 infected patients from

previous study;

30 randomly chosen for

each infection site,

total I50.

One French hospital.

(200I-2003)

36,836 patients ( 192 cases)

were admitted to one

general ICU in Belgium.

(1992-2002)

105 included cases, each matched with 2 controls

at one teaching hospital in

Rome, Italy

(Jan 1994-Jun 1995)
BSI, UTI, SSI, respiratory tract infection "and others" diagnosed $\geq 48$ hours after admission to ICU Patients with single-site nosocomial infection

Catheter-related bloodstream infection: positive culture results and clinical signs of sepsis

Bloodstream infection: isolated Diagnosed in $2 \%$ of pathogen(s) in the blood, plus screened patients one or more related symptom, $\geq 48$ hours after admission
14.5 NI episodes per 100 admissions

Not available

5.2 cases BSI per 1000 admissions, or I case per 1000 catheter-days
Not available

Not available

10 days attributable

Attributable LOS

19.1-19.8 days (mean), 13-15 days (median)
Mean excess cost:

AU $\$ 12,419 /$ case

SSI: attributable cost US $\$ 3529$ by generalized least squares, US $\$ 2852$ by propensity method. EM: US $\$ 3956$ by generalized least squares, US $\$ 3842$ by propensity method

US $\$ 10,015$ attributable cost per case

Attributable cost (mean $€$ ) by site of infection, UTI: $€ 574$ Surgical site: $€|8| 4$ Respiratory tract: $€ 242$ I Bloodstream: €953 Other: €1259

Attributable costs $€ 13,585$

Attributable $€ 15,413$ expenditure per case 
Table 3 (Continued)

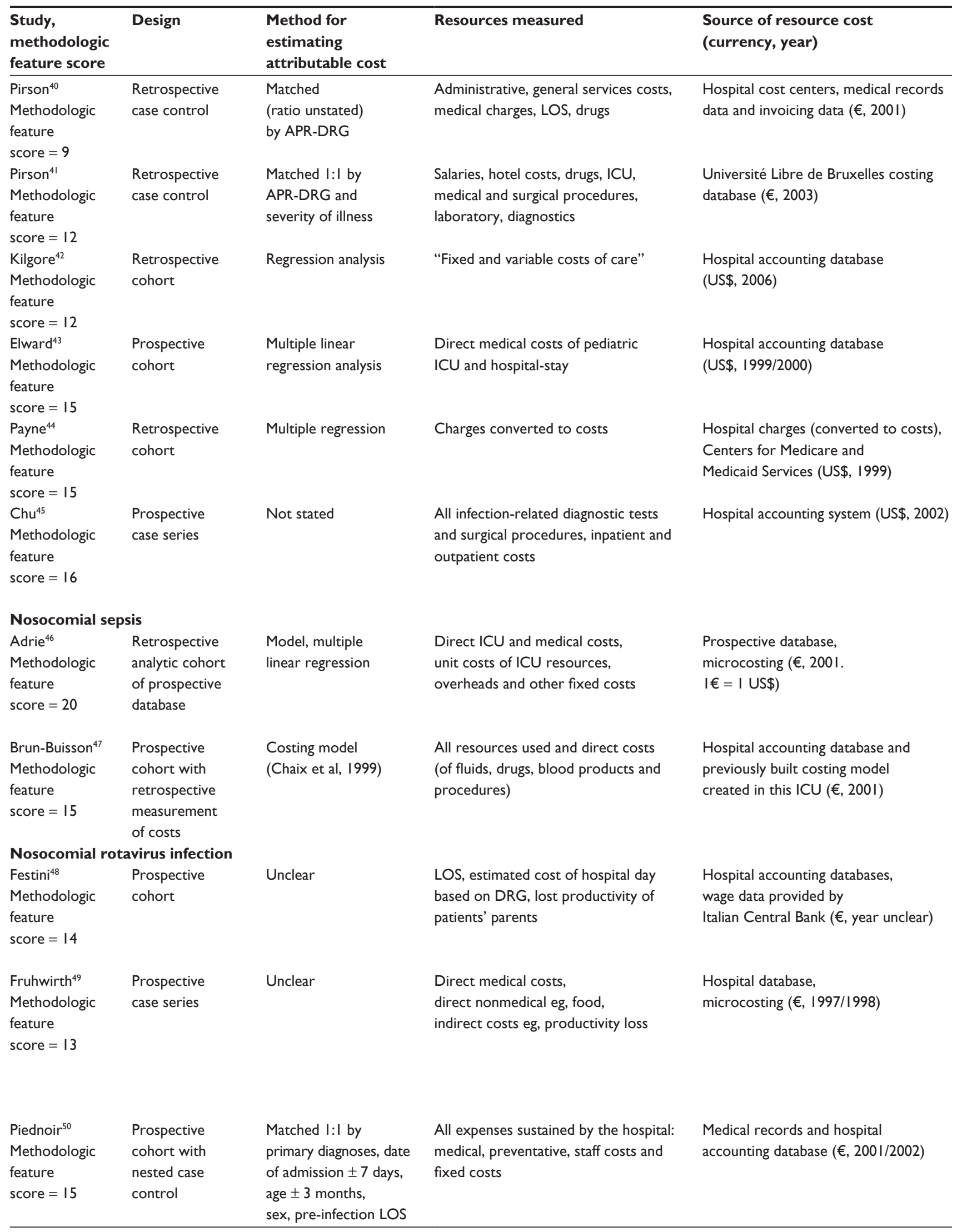




\begin{tabular}{|c|c|c|c|c|}
\hline $\begin{array}{l}\text { Sample population } \\
\text { (time horizon) }\end{array}$ & Case definition & Incidence & $\begin{array}{l}\text { Attributable } \\
\text { LOS }\end{array}$ & $\begin{array}{l}\text { Estimated } \\
\text { attributable cost }\end{array}$ \\
\hline $\begin{array}{l}46 \text { cases of HAB in one } \\
\text { Belgian hospital }(200 \mathrm{I})\end{array}$ & $\begin{array}{l}\text { An infection of bacteremia } \\
\text { developed } \geq 48 \text { hours after } \\
\text { admission }\end{array}$ & $0.56 \%$ incidence & 2I.I additional days & $\begin{array}{l}\text { Average attributable } \\
\text { costs: } € \mid 2,853\end{array}$ \\
\hline $\begin{array}{l}326 \text { cases in } 2003 \text { and } \\
277 \text { cases in } 2004 ; \\
3 \text { Belgian hospitals } \\
\text { (2003 and 2004) }\end{array}$ & $\begin{array}{l}\text { Cases were defined as } \\
\text { bacteremia that developed } \\
\geq 48 \text { hours after admission }\end{array}$ & $\begin{array}{l}\text { Incidence of HAB: } \\
\text { I.4\% and I. } 2 \% \text { in } \\
2003 \text { and } 2004\end{array}$ & $\begin{array}{l}\text { Attributable LOS: } \\
6.1 \text { days (ICU); } \\
30 \text { days (non-ICU) }\end{array}$ & $\begin{array}{l}\text { Mean additional cost of } \\
\text { HAB was } € 16,709\end{array}$ \\
\hline $\begin{array}{l}\text { I,355,647 admissions during } \\
69 \text { months from } 55 \text { hospital } \\
\text { databases (Mar 200I- } \\
\text { Jan 2006) }\end{array}$ & $\begin{array}{l}\text { Nosocomial BSIs, nonduplicate } \\
\text { isolate collected } \geq 3 \text { days after } \\
\text { admission }\end{array}$ & $\begin{array}{l}\text { Nosocomial BSIs } \\
\text { identified in } 0.93 \% \\
\text { of admissions }\end{array}$ & Not available & $\begin{array}{l}\text { Incremental costs: } \\
\text { US } \$ 19,427\end{array}$ \\
\hline $\begin{array}{l}91 \text { I admissions, including } \\
56 \text { case patients under } \\
\text { age I } 8 \text { in one US PICU } \\
\text { (Sep I, I999-May 3I, 2000) }\end{array}$ & $\begin{array}{l}\text { Bloodstream infections in PICU } \\
\text { patients, recognized pathogen } \\
\text { isolated from blood }>48 \text { hours } \\
\text { postadmission }\end{array}$ & $\begin{array}{l}\text { Rate of BSI: } \\
\text { I3.8 per } 1000 \\
\text { central venous } \\
\text { catheter days }\end{array}$ & Not available & $\begin{array}{l}\text { Attributable PICU direct } \\
\text { costs: US } \$ 39,219\end{array}$ \\
\hline $\begin{array}{l}2809 \text { VLBW infants in I7 NICUs, } \\
553 \text { with nosocomial BSI } \\
\text { (1998-1999) }\end{array}$ & $\begin{array}{l}\text { BSI after 3rd postnatal day, } \\
\text { with symptoms of infection } \\
\text { and } 5+\text { days antibiotic treatment } \\
\text { after diagnosis }\end{array}$ & $\begin{array}{l}\text { Nosocomial } \\
\text { BSI: } 19.7 \%\end{array}$ & $\begin{array}{l}\text { The mean additional } \\
\text { LOS of VLBW infants } \\
\text { with BSI was } 32.5 \text { days }\end{array}$ & $\begin{array}{l}\text { The mean attributable } \\
\text { cost was US } \$ 54,539\end{array}$ \\
\hline $\begin{array}{l}298 \text { patients with a } \\
\text { prosthetic implant and } \\
\text { S. aureus bacteremia (nosocomial } \\
\text { acquired) } \\
\text { (Sep 1994-Sep 2002) }\end{array}$ & $\begin{array}{l}\text { Positive blood culture for } \\
\text { S. aureus bacteremia, } \geq 72 \text { hours } \\
\text { postadmission, in a patient } \\
\text { with } \geq \text { I prosthetic implant }\end{array}$ & Not available & Mean 33 additional days & $\begin{array}{l}\text { Attributable cost per case: } \\
\text { US } \$ 67,439\end{array}$ \\
\hline $\begin{array}{l}\text { Of I } 698 \text { patients } \\
\text { hospitalized for more than } \\
48 \text { hours in } 6 \text { ICUs, } \\
340 \text { had sepsis. } \\
\text { (Apr 1997-Dec 2000) }\end{array}$ & $\begin{array}{l}\text { Severe sepsis: infection, } \\
\geq 2 \text { criteria for systemic } \\
\text { inflammatory response } \\
\text { syndrome and } \geq I \text { criterion } \\
\text { for organ dysfunction }\end{array}$ & $20.0 \%$ & Not available & US\$27,510 \\
\hline $\begin{array}{l}424 \text { patients in one Paris, France } \\
\text { ICU (1997-1998) }\end{array}$ & $\begin{array}{l}\text { Patients with sepsis, clinically or } \\
\text { microbiologically documented, } \\
\geq 48 \text { hours after admission }\end{array}$ & $\begin{array}{l}\text { ICU-acquired } \\
\text { sepsis: } 23 \%\end{array}$ & $\begin{array}{l}19 \text { additional days } \\
\text { compared to patients } \\
\text { with no sepsis }\end{array}$ & $\begin{array}{l}\text { Nosocomial cases } \\
\text { incurred average total } \\
\text { costs were } € 39,908 \\
\text { higher than patients with } \\
\text { no sepsis }\end{array}$ \\
\hline $\begin{array}{l}608 \text { children under } \\
30 \text { months of age in four } \\
\text { Italian hospitals } \\
(2006-2008)\end{array}$ & $\begin{array}{l}\text { Hospital-acquired, } \\
\text { positive rapid rotavirus testing }\end{array}$ & $\begin{array}{l}\text { Incidence of } \\
n R V I \text { was } 5.3 \%\end{array}$ & 1.7 attributable days & $\begin{array}{l}\text { National burden of } \\
\text { nosocomial rotavirus in } \\
\text { Italy, based on attributable } \\
\text { LOS, is estimated at } \\
€ 8,019,155\end{array}$ \\
\hline $\begin{array}{l}33 \text { cases of nosocomial rotavirus } \\
\text { infection in } \\
\text { children }<48 \text { months, } \\
\text { in Austria (Dec 1997- } \\
\text { May 1998) }\end{array}$ & $\begin{array}{l}\text { Rotavirus-positive diarrhea, } \\
\text { nosocomial if onset } \\
\text { was }>48 \text { hours after admission }\end{array}$ & $\begin{array}{l}\text { Risk for contracting } \\
\text { nRVI was } 2.59 \\
\text { per } 1000 \text { hospital } \\
\text { days during peak } \\
\text { rotovirus season } \\
\text { (Dec-May), } \\
<48 \text { months of age }\end{array}$ & Not available & Case cost average $€ 2442$ \\
\hline $\begin{array}{l}23 \text { cases matched I:I, } \\
\text { in one French pediatric } \\
\text { hospital (Dec I, 200I- }\end{array}$ & $\begin{array}{l}\text { Rotavirus-positive stool via } \\
\text { qualitative enzyme-linked } \\
\text { immunosorbent assay (ELISA) }\end{array}$ & $\begin{array}{l}\text { Attack rate: } 6.6 \% \\
\text { Incidence: I } 5.8 \text { per } \\
\text { I000 hospital days }\end{array}$ & 4.9 additional days & $\begin{array}{l}\text { Mean attributable cost } \\
\text { due to nosocomial } \\
\text { rotavirus infection: } € 1930\end{array}$ \\
\hline
\end{tabular}


Table 3 (Continued)

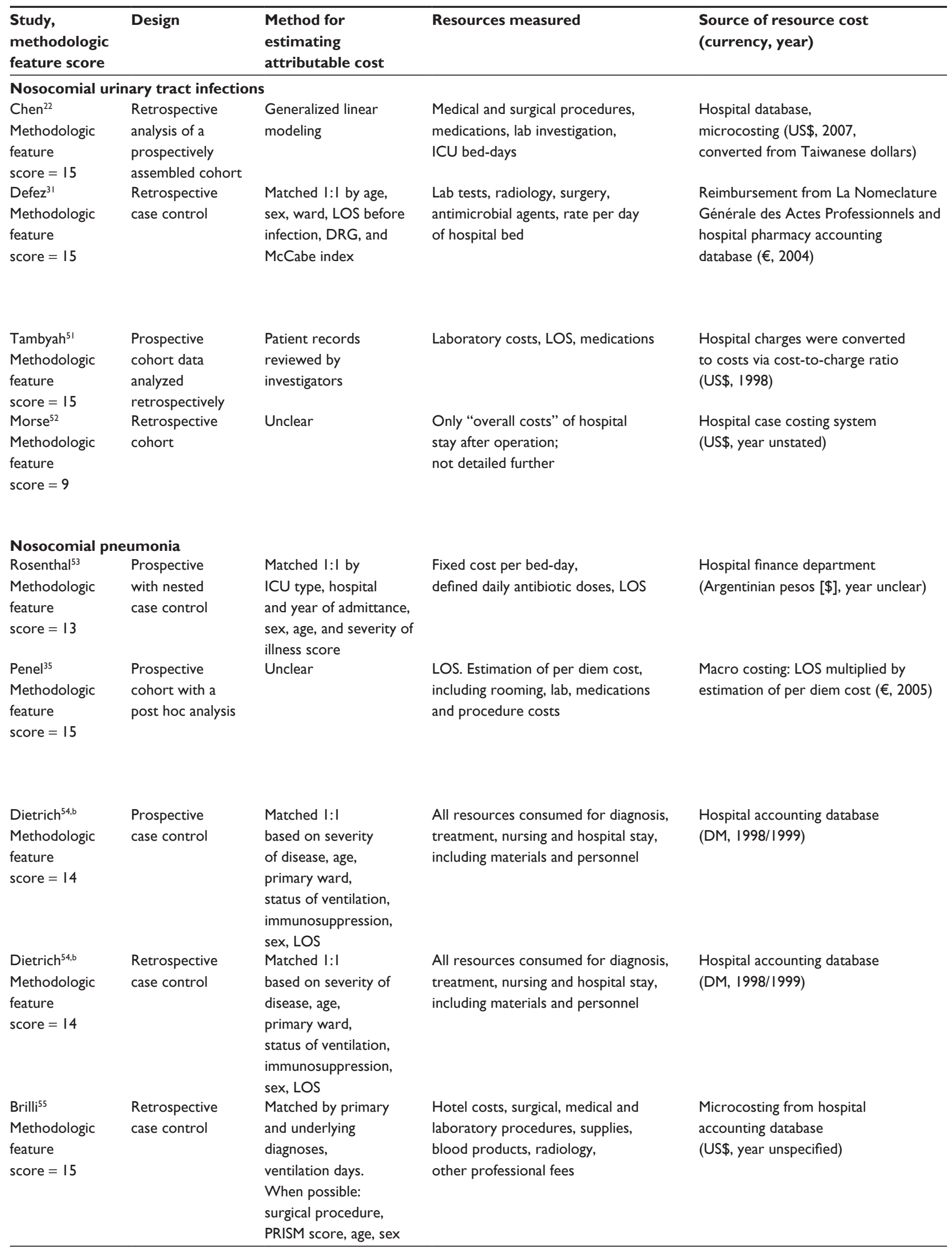




\section{Sample population} (time horizon)

Case definition Incidence

Incidence

Attributable

LOS

Not available

I4.5 NI episodes per 100 admissions infection "and others" diagnosed $\geq 48$ hours after admission to ICU Patients with single-site nosocomial infection

1703 infected patients from previous study;

30 randomly chosen

for each infection site,

total I 50.

One French hospital. (200I-2003)

I 497 catheterized patients in one US university

hospital, of which 223

had UTI (1997-1998)

I 18 bowel surgery patients aged "Never events:" hospital-

65 to 79 , and 33 aged $>80$,

with Medicare in one hospital;

total of 64 patients experience

a "never event"

(Jan 2008-Mar 2009)

\section{7 case patients}

(pneumonia),

307 control patients

in 3 hospitals over

5 years (1998-2002)

261 head/neck cancer

surgery patients in

one hospital

SSI: 94

PP: 34

SSI and PP: 13

(Jan 1997-Dec 1999)

48 cases and 66 controls

(resulting in 29 matched

pairs) in one German

teaching hospital,

5 ICUs (May 1998-

Mar 1999)

37 matched pairs in one

German teaching hospital,

admitted to one of

2 neurosurgical wards

(Feb 1997-Dec 1998)

I3 case patients matched

to control patients I:I

in one pediatric ICU

(FY 2005-FY 2007)
Nosocomial UTI, defined as new bacteriuria or funguria exceeding $10^{3} \mathrm{CsFU} / \mathrm{mL}$ acquired complications that are not reimbursed by Medicare

$14.9 \%$ of catheterized patients

$42.4 \%$ of study patients experienced a "never event"

Nosocomial pneumonia according to definition from the CDC

$5.79 \%$ developed nosocomial

pneumonia

Based on the CDC 1992; SSI, PP

SSI: $36 \%$

PP: 13\%

SSI and PP: $5 \%$

Not available

Nosocomial pneumonia, diagnosed according to the criteria of the CDC

Nosocomial pneumonia, diagnosed according to the criteria of the CDC

Pediatric ICU patients with VAP
7.8 cases per 1000 ventilator days in FY 2005
5.00 additional ventilation days, 6.55 additional days in ICU and

7.40 additional

days in hospital

5.00 additional ventilation days, I4.03 additional days in ICU and 10.14 additional days in hospital excess days

8.7 attributable days Attributable VAP costs per patient: US\$5I,I57.

Attributable cost per hospital perspective perspective
Attributable cost (mean $€$ ) by site of infection UTI: $€ 574$

Surgical site: $€ 1814$ Respiratory tract: $€ 2421$ Bloodstream: €953 Other: €I259

Average attributable treatment cost: US\$589

Catheter-related UTI: US\$14,300 extra costs; vascular catheter infection: US\$16,400 extra costs

Mean attributable cost for cases was ARS $\$ 2255$

SSI: $€ I 6,000$ increase in mean direct medical costs PP: $€ I 7,000$

Both SSI and PP: $€ 35,000$ case: DM 14,606 from the

Attributable cost per case: DM 29,6I 0 from hospital 
Table 3 (Continued)

\begin{tabular}{|c|c|c|c|c|}
\hline $\begin{array}{l}\text { Study, } \\
\text { methodologic } \\
\text { feature score }\end{array}$ & Design & $\begin{array}{l}\text { Method for } \\
\text { estimating } \\
\text { attributable cost }\end{array}$ & Resources measured & $\begin{array}{l}\text { Source of resource cost } \\
\text { (currency, year) }\end{array}$ \\
\hline \multicolumn{5}{|c|}{ Nosocomial respiratory tract infection } \\
\hline $\begin{array}{l}\text { Defez }{ }^{31} \\
\text { Methodologic } \\
\text { feature } \\
\text { score }=15\end{array}$ & $\begin{array}{l}\text { Retrospective } \\
\text { case control }\end{array}$ & $\begin{array}{l}\text { Matched I:I by } \\
\text { age, sex, ward, } \\
\text { LOS before infection, } \\
\text { DRG, and } \\
\text { McCabe index }\end{array}$ & $\begin{array}{l}\text { Lab tests, radiology, surgery, } \\
\text { antimicrobial agents, rate per day } \\
\text { of hospital bed }\end{array}$ & $\begin{array}{l}\text { Reimbursement from La Nomeclature } \\
\text { Générale des Actes Professionnels and } \\
\text { hospital pharmacy accounting } \\
\text { database }(€, 2004)\end{array}$ \\
\hline $\begin{array}{l}\text { Chen }^{22} \\
\text { Methodologic } \\
\text { feature } \\
\text { score = } 15\end{array}$ & $\begin{array}{l}\text { Retrospective } \\
\text { analysis of a } \\
\text { prospectively } \\
\text { assembled cohort }\end{array}$ & $\begin{array}{l}\text { Generalized linear } \\
\text { modeling }\end{array}$ & $\begin{array}{l}\text { Medical and surgical procedures, } \\
\text { medications, lab investigation, } \\
\text { ICU bed-days }\end{array}$ & $\begin{array}{l}\text { Hospital database, } \\
\text { microcosting (US\$, 2007, } \\
\text { converted from Taiwanese dollars) }\end{array}$ \\
\hline $\begin{array}{l}\text { McCartney } \\
\text { Methodologic } \\
\text { feature } \\
\text { score }=15\end{array}$ & Case control & $\begin{array}{l}\text { Matched I:I by } \\
\text { age, principal } \\
\text { discharge diagnosis, } \\
\text { same RSV season, } \\
\text { number of } \\
\text { secondary diagnoses }\end{array}$ & Direct medical costs & $\begin{array}{l}\text { Hospital accounting database } \\
\text { (US\$, 1996) }\end{array}$ \\
\hline \multicolumn{5}{|c|}{ Miscellaneous nosocomial infections } \\
\hline $\begin{array}{l}\text { Bou }^{57} \\
\text { Methodologic } \\
\text { feature } \\
\text { score }=13\end{array}$ & $\begin{array}{l}\text { Retrospective } \\
\text { case series }\end{array}$ & $\begin{array}{l}\text { Multiple linear } \\
\text { regression analysis }\end{array}$ & $\begin{array}{l}\text { ICU hospital costs only: treatments } \\
\text { and diagnostic procedures }\end{array}$ & $\begin{array}{l}\text { Hospital finance department, } \\
\text { microcosting ( } € \text {, year unspecified) }\end{array}$ \\
\hline $\begin{array}{l}\text { Fretz }^{58} \\
\text { Methodologic } \\
\text { feature } \\
\text { score }=16\end{array}$ & $\begin{array}{l}\text { Retrospective } \\
\text { case series }\end{array}$ & Unclear & $\begin{array}{l}\text { Revenue loss, nursing, diagnostic } \\
\text { procedures, pharmacy, costs of } \\
\text { creating an isolation ward }\end{array}$ & $\begin{array}{l}\text { Hospital department- } \\
\text { specific costs ( } €, \text { year unspecified) }\end{array}$ \\
\hline $\begin{array}{l}\text { Zingg } \\
\text { Methodologic } \\
\text { feature } \\
\text { score }=17\end{array}$ & $\begin{array}{l}\text { Retrospective } \\
\text { case control }\end{array}$ & $\begin{array}{l}\text { Matched I:2 by } \\
\text { age, sex, } \\
\text { LOS, underlying } \\
\text { disease category }\end{array}$ & $\begin{array}{l}\text { Direct impact on hospital resources: } \\
\text { loss of revenue, additional } \\
\text { microbiological diagnosis }\end{array}$ & $\begin{array}{l}\text { Hospital database, } \\
\text { microcosting (US\$, 2002) }\end{array}$ \\
\hline $\begin{array}{l}\text { Anil }{ }^{60} \\
\text { Methodologic } \\
\text { feature } \\
\text { score }=12\end{array}$ & $\begin{array}{l}\text { Retrospective } \\
\text { case control }\end{array}$ & $\begin{array}{l}\text { Matched I:I } \\
\text { based on birth } \\
\text { weight } \pm 10 \% \text {, sex, } \\
\text { gestational } \\
\text { age } \pm 2 \text { weeks, } \\
\text { ventilation, } \\
\text { anti-microbial therapy, } \\
\text { use of CVC/TPN }\end{array}$ & $\begin{array}{l}\text { Charges per patient and actual } \\
\text { financial burden of outbreak; } \\
\text { not detailed further }\end{array}$ & $\begin{array}{l}\text { Hospital discharge abstracts } \\
\text { via the hospital's central } \\
\text { finance service (US\$, assumed 2005) }\end{array}$ \\
\hline $\begin{array}{l}\text { Baggett }^{61} \\
\text { Methodologic } \\
\text { feature } \\
\text { score }=18\end{array}$ & $\begin{array}{l}\text { Retrospective } \\
\text { case series }\end{array}$ & $\begin{array}{l}\text { Interviews with } \\
\text { hospital staff and } \\
\text { review of contact } \\
\text { tracing logs }\end{array}$ & $\begin{array}{l}\text { Direct costs: personnel time, } \\
\text { laboratory and medication costs; } \\
\text { Indirect: hospital staff furloughs }\end{array}$ & $\begin{array}{l}\text { Hospital database, } \\
\text { microcosting (US\$, 2004) }\end{array}$ \\
\hline $\begin{array}{l}\text { Spearing }{ }^{62} \\
\text { Methodologic } \\
\text { feature } \\
\text { score = } 15\end{array}$ & $\begin{array}{l}\text { Retrospective } \\
\text { cohort }\end{array}$ & Unclear & $\begin{array}{l}\text { Direct costs including medical costs, } \\
\text { outbreak investigation, } \\
\text { lost productivity costs, and misc }\end{array}$ & $\begin{array}{l}\text { Medical records data and } \\
\text { Medicare costs (AU\$, 1996) }\end{array}$ \\
\hline $\begin{array}{l}\text { Wilson }^{63} \\
\text { Methodologic } \\
\text { feature } \\
\text { score }=13\end{array}$ & $\begin{array}{l}\text { Retrospective } \\
\text { with nested } \\
\text { case control }\end{array}$ & $\begin{array}{l}\text { Matched I:I to } \\
\text { controls with } \geq 20 \% \\
\text { total body surface } \\
\text { burns }\end{array}$ & Hospital charges converted to costs & $\begin{array}{l}\text { Hospital finance department; } \\
\text { unclear costing methods (US\$, 200I) }\end{array}$ \\
\hline
\end{tabular}




\section{Sample population} (time horizon)
Case definition

I 703 infected patients from

previous study;

30 randomly chosen

for each infection site,

total 150.

One French hospital.

(200I-2003)

40 I NIs in 320 of 2757 patients,

in 4 ICUs in one hospital in

Taiwan (2003-2004)

All patients admitted to

one Philadelphia pediatric

hospital over 8 RSV

seasons (1988-1996)
Patients with single-site nosocomial infection

diagnosed $\geq 48$ hours after

admission to ICU

Nosocomial RSV infection
Not available

I4.5 NI episodes

per 100 admissions

Incidence

88 nosocomial

RSV cases out of

90, 174 patients

\section{Attributable}

LOS
Estimated

attributable cost
Not available

Attributable LOS for nosocomial RSV

was 7.8 days
Attributable cost (mean $€$ ) by site of infection, UTI: $€ 574$

Surgical site: $€ 1814$ Respiratory tract: $€ 242$ I Bloodstream: €953 Other: €। 259

US\$10,0I5 attributable cost per case

Mean attributable cost to hospital per RSV NI was US\$9419/case

\author{
67 ICU patients during a \\ $P$. aeruginosa outbreak at \\ one ICU in Spain (Jul- \\ Sep 2003) \\ 90 infected patients and \\ staff of an Austrian hospital \\ during a norovirus \\ outbreak (Dec 2006- \\ Feb 2007) \\ 16 case patients and \\ 32 control patients during a \\ norovirus outbreak \\ (200I and 2002) \\ 22 cases in one Turkish NICU, \\ drug-resistant \\ S. typhimurium outbreak \\ (Mar 15-29, 2005)
}

Any patient who developed the infection after $\geq 48$ hours mechanical ventilation

Positive stool specimen for norovirus $\geq 48$ hours

following admission

A person who developed acute Attack rate 13.9\%

diarrhea, nausea and vomiting among patients and

during the norovirus outbreak $29.5 \%$ among health care workers

Positive stool/rectal swab or fluid culture for S. typhimurium
Incidence of outbreak associated with pseudomonas infection; 17/67

Not applicable
38 additional days

Not available

Not available

9.8 additional days
$€ 18,408$ average extra ICU costs per case patient

The total cost of the outbreak for the Department of Internal Medicine was $€ 80,138$

US\$2452 per case (derived from US $\$ 40,675$ total direct outbreak costs $\div 16$ case patients) US\$1081.84 more charges per case compared to control

$\begin{array}{ll}\begin{array}{l}\text { Two hospitals experiencing } \\ \text { a nosocomial pertussis outbreak } \\ \text { (Jul 25- }\end{array} & \begin{array}{l}\text { A cough illness lasting } \\ \text { Sep I5, 2004) } \\ \text { of whooping with symptoms } \\ \text { isolation of B. pertussis or } \\ \text { confirmed by PCR or culture } \\ 52 \text { cases in a }\end{array} \\ \begin{array}{l}\text { Not detailed; cases of Salmonella } \\ \text { complex during an } \\ \text { outbreak of Salmonella } \\ \text { (Dec I996) }\end{array} & \\ 34 \text { burn patients } & \\ \text { (Jan-Dec 2004) } & \text { Hospital-acquired nosocomial }\end{array}$

Two hospitals experiencing

Incidence was 10/1475 persons exposed

$16 \%$ of 217 burn patients acquired MDRAB infection
Not available

Not available

I I additional days
Attributable cost per nosocomial case, Hospital A: US\$43,893 Hospital B: US\$30,282

AU\$2308 (US\$1827) per case (total outbreak cost AU $\$ 120,000$ or US $\$ 95,000 \div 52$ cases)

Mean additional cost: US\$98,575 
Table 3 (Continued)

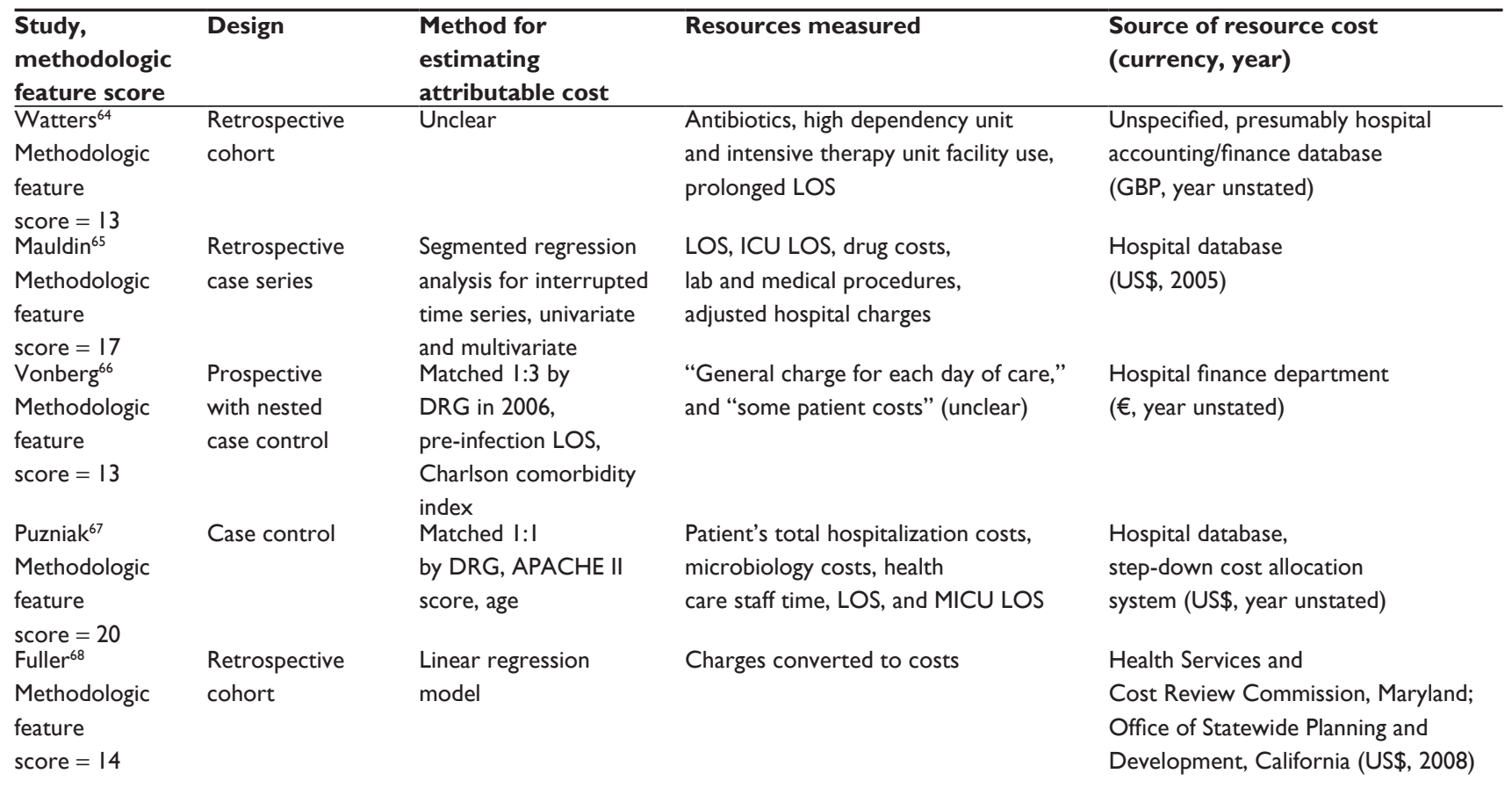

Nosocomial venous thromboembolism

$\begin{array}{lll}\begin{array}{l}\text { Maprini69 } \\ \text { feature }\end{array} & \begin{array}{l}\text { Decision } \\ \text { analysis (Markov) }\end{array} & \text { Univariate analysis } \\ \text { score }=18 & & \\ \begin{array}{l}\text { MacDougall }{ }^{70} \\ \text { Methodologic }\end{array} & \begin{array}{l}\text { Retrospective } \\ \text { observational } \\ \text { feature }\end{array} & \begin{array}{l}\text { Linear model with } \\ \text { log-link function and }\end{array} \\ \end{array}$

Patient care protocols, health care staff time, diagnostic tests, supplies, hospitalizations, procedures

Treatment strategy, length of hospital stay, physician office, emergency room, outpatient claims, ancillary services, pharmacy utilization
Literature data (US\$, year unstated)

Actual health care plan payments

for services only

score $=16$

\begin{tabular}{|c|c|c|}
\hline \multicolumn{3}{|c|}{ Nosocomial related falls } \\
\hline $\begin{array}{l}\text { Methodologic } \\
\text { feature } \\
\text { score = } 12\end{array}$ & & \\
\hline $\begin{array}{l}\text { Oliver }^{72} \\
\text { Methodologic } \\
\text { feature } \\
\text { score = } 13\end{array}$ & Case series & N/A \\
\hline $\begin{array}{l}\text { Nurmi }{ }^{73} \\
\text { Methodologic } \\
\text { feature } \\
\text { score = } 13\end{array}$ & $\begin{array}{l}\text { Prospective } \\
\text { cohort }\end{array}$ & Unclear \\
\hline
\end{tabular}

Operation procedures, nonoperative treatment, LOS

Legal payments

Emergency room visits, outpatient visits, LOS, radiology
Southport and Ormskirk Hospital

Risk Management Department; Hospital Finance Department (GBP, year unspecified)

NHS Litigation Authority Database of clinical negligence claims (GBP, year unspecified)

Hospital accounting database (€, 1999)

Notes: a6I studies in total, of which three reported outcomes for more than one type of infection, therefore the total listed in Table 2 is 68; bietrich, 2002 is one paper detailing two different studies. The studies were separated in this table for clarity; *only gives incidence of PTS, PEs given post-surgical DVT.

Abbreviations: ADE, adverse drug event; AE, adverse event; APACHE, Acute Physiology and Chronic Health Evaluation; APR, all patient refined; ARS, Argentine peso; AU, Australian; BSI, blood stream infection; CABG, coronary artery bypass graft; CDAD, Clostridium difficile-associated disease; CDC, Centers for Disease Control and Prevention; CVC/TPN, central venous catheter/total parenteral nurtition; DM, Deutsche Mark; DRG, disease-related group; DVT, deep vein thrombosis; EM, endometriosis; FY, financial year; GBP, Great Britain Pound; HAB, hospital-acquired bacteremia; HAC, hospital-acquired complication; HAI, hospital-acquired infection; ICU, intensive care unit; JP, Japanese; LOS, length of stay; MICU, medical intensive care unit; MDRAB, multidrug-resistant Acinetobacter bowmanii; MRSA, methicillin-resistant Staphylococcus aureus; NHS, National Health Service; NI, nosocomial infection; NICU, neonatal ICU; NIM, Nosocomial Infection Marker; NNIS, National Nosocomial Infections Surveillance; nRVI, nosocomial rotovirus infection; NZ, New Zealand; PE, pulmonary embolism; PICU, perinatal ICU; PP, postoperative pneumonia; PTS, postthrombotic syndrome; RSV, respiratory syntactical virus; SCl, spinal cord injury; SSI, surgical site infection; SSTI, skin soft tissue infection;TSI,Transition Systems Incorporated; UTI, urinary tract infection;VAP, ventilator-associated pneumonia;VLBW, very low birth weight; VRE, vancomycin-resistant Enterococcus. 


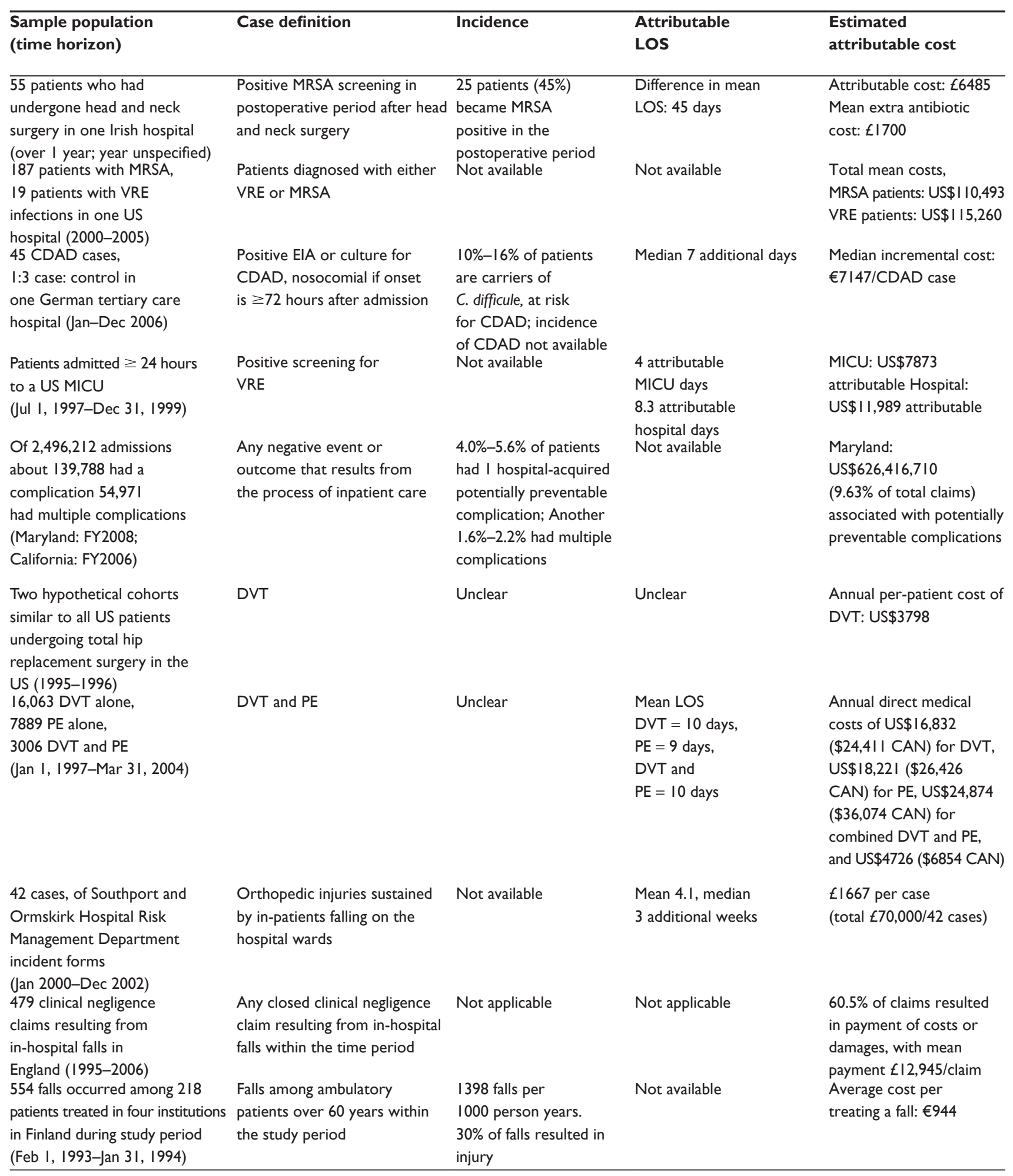




\section{Nosocomial venous thromboembolism (VTE)}

We identified two burden studies published since 2000. One study focused on nosocomial deep vein thrombosis after hip replacement surgery. ${ }^{69}$ The cost of deep vein thrombosis was modeled in patients undergoing total hip replacement surgery, with Markov decision and univariate analyses. The article reported the annual per-patient cost of deep vein thrombosis to be $\$ 4676$. Also provided are the discounted lifetime costs of $\$ 3779$, as well as costs specific to deep vein thrombosis-related complications, namely post-thrombotic syndrome with ulcer (\$4700) and pulmonary embolism (\$8131). A retrospective US study of deep vein thrombosis $(\mathrm{n}=15,679)$, pulmonary embolism $(n=7653)$ and post-thrombotic syndrome $(n=624)$ found annual attributable direct medical costs of $\$ 19,430$ for deep vein thrombosis, $\$ 21,033$ for pulmonary embolism, $\$ 28,713$ for combined deep vein thrombosis, and pulmonary embolism, and $\$ 5455$ for patient safety target. This study did not explicitly distinguish cases of nosocomial deep vein thrombosis, but $78 \%$ of the study cohort had abdominal or orthopedic surgery prior to the index venous thrombosis event. ${ }^{70}$

\section{Nosocomial falls}

We reviewed three burden-of-illness studies related to nosocomial falls. Two studies were case series. ${ }^{71,72}$ The third study had a prospective cohort design. ${ }^{73}$ One study ${ }^{73}$ identified cases only in patients aged over 60 years. One study focused on legal compensation rather than hospitalrelated costs, ${ }^{72}$ and neither of the other two articles ${ }^{71,73}$ clearly stated what methods were used for determining attributable costs. There was one additional case control study reported attributable length of stay, but not costs. ${ }^{74}$

Oliver found that $60.5 \%$ of legal claims related to in-hospital falls resulted in payment of costs or damages, with mean payment of $\$ 25,793 .{ }^{72}$ Nurmi provided the cost per treating an in-hospital fall, estimated at $\$ 1359 .{ }^{73}$ The third study did not describe costs per case or per fall, but did provide the total estimated attributable cost of all cases included in the study; dividing by the provided number of cases yields a crude estimate of $\$ 3230$ per case. ${ }^{71}$

We did not find any eligible studies for the following target conditions: nosocomial pressure ulcers, wrong site surgery, retained surgical foreign bodies, contrast inducted nephropathy.

\section{Discussion}

We found that $61 \%$ of published studies on the economic burden of patient safety targets in acute care describe or report no clear costing methodology. Among the remaining 61 studies (39\%), which did report costing methodology, there were wide variations in methodologic features and methods for attributing costs. These studies report wide estimates of the economic burden of patient safety in acute care. For example, the attributable costs of patient safety targets ranged from $\$ 2000$ to $\$ 200,000$. In general hospital populations, the cost per case of hospital-acquired infection ranged from $\$ 2132$ to $\$ 15,018$. Nosocomial bloodstream infections were associated with costs ranging from $\$ 2604$ to $\$ 22,414$. We also found no adequate economic burden data for important patient safety, such as wrong site surgery, retained surgical foreign bodies, contrast-induced nephropathy, and acute care-acquired pressure ulcers.

Our results are consistent with the few prior reviews of the economic burden of patient safety in the acute care setting. A 2005 review identified 165 articles that included an economic analysis as an objective, but $35 \%$ of these articles provided no economic analysis, and $25 \%$ provided no primary economic data. The remaining studies had significant gaps in their costing methodology, and only $16 \%$ conducted sensitivity analyses that could address these limitations. ${ }^{75}$ Another review of comparative economic evaluations of patient safety programs identified 40 studies published between 2001 and 2004, none of which provided sufficient information about both the cost of the prevention program and the cost of the patient safety being targeted. ${ }^{76}$ A 1999 study estimated the economic burden of patient safety in Utah and Colorado at $\$ 1,442,024$ per event (1996 US dollars). This early estimate is much higher than estimates in our systematic review, because the 1999 study evaluated not just direct acute care costs, but also outpatient direct health care costs after the event, as well as indirect (societal) costs such as lost workforce productivity up to age 75 years. None of the adverse event studies in our review considered this broad range of costs for a prolonged time horizon. ${ }^{77}$

Our findings, in conjunction with these prior reviews, indicate that greater attention is needed to the methodologic standards for evaluating the economic burden of patient safety in the acute care setting. Better knowledge of the economic burden of patient safety will inform decisions about health policy, patient safety research programs, and improvement priorities. High quality economic burden studies are an essential component of comparative economic analyses, such as cost effectiveness studies. Most of the studies we identified considered only the acute care hospital economic perspective, but the economic perspective should extend beyond the acute care hospital, as it has been estimated that $22 \%-66 \%$ of the 
economic burden of patient safety in acute care are borne by the hospital. ${ }^{78,79}$ Economic burden studies for patient safety should explicitly consider cost categories, legal, marketing and operational perspectives (direct or indirect), and time frames (including short- and long-term effects). There are also important methodologic considerations when attributing costs to patient safety, rather than the patient's underlying condition. These considerations include the reliability of data sources used to identify patient safety, the adequacy of methods to control for confounding factors such as comorbidity and severity of illness, and the appropriateness of estimation methods including the incorporation of adverse event timing, matching methods, and regression modeling. ${ }^{80}$ Differential timing of the occurrence of patient safety can lead to wide estimates of attributable costs. ${ }^{81-83}$

Our review has several important limitations. First, we focused on studies published between 2000 and 2010 and indexed in MEDLINE. Studies outside of our search strategy may contain potentially useful data. For example, we did not include a 2010 study by the Society of American Actuaries because it was not indexed in MEDLINE. ${ }^{84}$ However, our finding that $61 \%$ of studies provide no or limited costing methodology would be unchanged by the inclusion of a few additional studies. Second, we focused only on patient safety targets in the acute care setting. We did not include studies of patient safety targets from other settings, such as community or chronic care. Third, we did not evaluate the interrater reliability of our methodologic reviews. Our review method was designed to yield higher methodologic ratings, as we always took the higher rating of the two reviewers, yet we still identified a significant lack of methodologic features. Fourth, we arbitrarily assigned one point for each methodologic feature, so that we could report a simple summary measure of methodologic features. However, we recognize that methodologic features are not necessarily equally weighted. Finally, there was heterogeneity in study methods. Variability in costing and methodologic features made it impossible to generate summary estimates of economic burden for all patient safety targets.

In summary, the burden of patient safety targets ranged from as little as $\$ 2000$ to $\$ 200,000$ in hospitalized individuals to $\$ 600$ million at a population level. These results are dependent on the resources and costs included in the analysis. We found that the majority of published studies on the economic burden of patient safety targets in acute care described no costing methodology. The methodologic quality of the remaining studies was moderate, but there were wide variations in methodologic quality and methods for attributing costs. Greater attention is needed to the methodologic standards for evaluation of economic burden. This study highlights the limitations in the methods required to conduct economic evaluations in patient safety. Such limitations make decision-making regarding the adoption of patient safety initiatives difficult. The identification of limitations will allow for focused work on their improvement and will allow for the development of guidelines for future economic evaluation in patient safety.

\section{Contributions}

Dr Nicole Mittmann, Ms Marika Koo, Dr Nick Daneman, Dr Andrew McDonald, Dr Michael Baker, Dr Anne Matlow, Dr Murray Krahn, Dr Kaveh Shojania, and Dr Edward Etchells all had substantial contributions to the concept and design, acquisition of data, or analysis and interpretation of data. All authors contributed to the draft or revision of the article in a critical manner and they all gave final approval of the version being submitted.

\section{Acknowledgments}

The authors acknowledge Ms Peggy Kee and Ms Evelyn Worthington for their administrative and data analysis, and Dr William Geerts, Dr Damon Scales, and Dr Andrew Simor for their assistance in the study design. This work was funded by an unrestricted grant from the Canadian Patient Safety Institute.

\section{Disclosure}

The authors report no conflicts of interest in this work.

\section{References}

1. Landrigan CP, Parry GJ, Bones CB, Hackbarth AD, Goldmann DA, Sharek PJ. Temporal trends in rates of patient harm resulting from medical care. N Engl J Med. 2010;363(22):2124-2134.

2. Classen DC, Resar R, Griffin F, et al. "Global trigger tool" shows that adverse events in hospitals may be ten times greater than previously measured. Health Aff (Millwood). 2011;30(6):581-589.

3. Canadian Agency for Drugs and Technologies in Health. Guidelines for the economic evaluation of health technologies: Canada [Internet]. 2006. 3rd ed. Ottawa, ON: Canadian Agency for Drugs and Technologies in Health; vii, 46, A17 p. [cited Oct 20]. Available from: http://www.cadth.ca/media/ pdf/186_EconomicGuidelines_e.pdf. Accessed September 30, 2011.

4. Mittmann N, Evans WK, Rocchi A, et al. Addendum to CADTH's guidelines for the economic evaluation of health technologies: specific guidance for oncology products. Ottawa: Canadian Agency for Drugs and Technologies in Health. 2009.

5. Gabriel S, Drummond M, Maetzel A, et al. OMERACT 6 Economics working group report: a proposal for a reference case for economic evaluation in rheumatoid arthritis. J Rheumatol. 2003;30(4):886-890.

6. Drummond MF, Jefferson TO. Guidelines for authors and peer reviewers of economic submissions to the BMJ. BMJ. 1996;313:275-283.

7. Drummond MF, O'Brien B, Stoddart GL, et al. Methods for the Economic Evaluation of Health Care Programmes. 2nd ed. Oxford. UK: Oxford Medical Publications; 1997.

8. Shojania KG, Duncan BW, McDonald KM, Wachter RM. Making health practices safer: a critical analysis of patient safety practices. 2001. Report No: Evidence Report/Technology Assessment No 43. 
9. National Surgical Quality Improvement Program. Program Specifics: Data Analysis and Reporting. 2006. Available from: http://www.acsnsqip.org/ main/programspecs/program_reporting jsp. Accessed September 30, 2011.

10. World Health Organization. Patient Safety: Implementing Change. 2011. Available from: http://www.who.int/patientsafety/implementation/en/. Accessed September 30, 2011.

11. Bank of Canada. Daily currency converter. 2011. Available from: http://www.bankofcanada.ca/rates/exchange/daily-converter/. Accessed September 30, 2011.

12. United States Department of Labor BoLS. CPI Inflation Calculator. 2011. Available from: http://www.bls.gov/data/inflation_calculator.htm. Accessed September 30, 2011.

13. Hoonhout LH, de Bruijne MC, Wagner C, et al. Direct medical costs of adverse events in Dutch hospitals. BMC Health Serv Res. 2009;9:27-37.

14. Ehsani JP, Jackson T, Duckett SJ. The incidence and cost of adverse events in Victorian hospitals 2003-2004. Med J Aust. 2006;184(11): 551-555.

15. New PW, Jackson T. The costs and adverse events associated with hospitalization of patients with spinal cord injury in Victoria, Australia. Spine. 2010;35(7):796-802.

16. Pappas SH. The cost of nurse-sensitive adverse events. $J$ Nurs Adm. 2008;38(5):230-236.

17. Morris JA, Carrillo Y, Jenkins JM, et al. Surgical adverse events, risk management, and malpractice outcome: Morbidity and mortality review in not enough. Ann Surg. 2003;237(6):844-852.

18. Aoki N, Uda K, Ohta S, Kiuchi T, Fukui T. Impact of miscommunication in medical dispute cases in Japan. Int J Qual Health Care. 2008;20(5): 358-362.

19. Kaushal R, Bates DW, Franz C, Soukup JR, Rothschild JM. Costs of adverse events in intensive care units. Crit Care Med. 2007;35(11): 2479-2483.

20. Senst BL, Achusim LE, Genest RP, et al. Practical approach to determining costs and frequency of adverse drug events in a health care network. Am J Health-Syst Pharm. 2001;58(12):1126-1132.

21. Chen YY, Chou YC, Chou P. Impact of nosocomial infection on cost of illness and length of stay in intensive care units. Infect Control Hosp Epidemiol. 2005;26(3):281-287.

22. Chen YY, Wang FD, Liu CY, Chou P. Incidence rate and variable cost of nosocomial infections in different types of intensive care units. Infect Control Hosp Epidemiol. 2009;30(1):39-46.

23. Roberts RR, Scott D, Cordell R, et al. The use of economic modeling to determine the hospital costs associated with nosocomial infections. Clin Infect Dis. 2003;36(11):1424-1432.

24. Kilgore ML, Ghosh K, Beavers M, Wong DY, Hymel PA, Brossette SE. The costs of nosocomial infections. Med Care. 2008;46(1): 101-104.

25. Esatoglu AE, Agirbas I, Onder OR, Celik Y. Additional cost of hospitalacquired infection to the patient: a case study in Turkey. Health Serv Manage Res. 2006;19(3):137-143.

26. Sheng WH, Wang JT, Lu DCT, Chie WC, Chen YC, Chang SC. Comparative impact of hospital-acquired infections on medical costs, length of hospital stay and outcome between community hospitals and medical centres. $J$ Hosp Infect. 2005;59(3):205-214.

27. Plowman R, Graves N, Griffin MAS, et al. The rate and cost of hospital-acquired infections occurring in patients admitted to selected specialties of a distract general hosptial in England and the national burden imposed. J Hosp Infect. 2001;47(198):209.

28. Lee J, Imanaka Y, Sekimoto M, et al. Risk-adjusted increases in medical resource utilization associated with health care-associated infections in gastrectomy patients. J Eval Clin Pract. 2010;16: $100-106$.

29. Mahieu LM, Buitenweg N, Beutels P, De Dooy JJ. Additional hospital stay and charges due to hospital-acquired infections in a neonatal intensive care unit. J Hosp Infect. 2001;47(3):223-229.

30. Graves N, Nicholls TM, Morris AJ. Modeling the costs of hospitalacquired infections in New Zealand. Infect Control Hosp Epidemiol. 2003;24(3):214-223.
31. Defez C, Fabbro-Peray P, Cazaban M, Boudemaghe T, Sotto A, Daures JP. Additional direct medical costs of nosocomial infections: an estimation from a cohort of patients in a French university hospital. J Hosp Infect. 2008;68(2):130-136.

32. Weber WP, Zwahlen M, Reck S, Feder-Mengus C, Widmer AF, Marti WR. Economic burden of surgical site infections at a European university hospital. Infect Control Hosp Epidemiol. 2008;29(7):623-629.

33. Whitehouse JD, Friedman D, Kirkland KB, Richardson WJ, Sexton DJ. The impact of surgical-site infections following orthopedic surgery at a community hospital and a university hospital: adverse quality of life, excess length of stay, and extra cost. Infect Control Hosp Epidemiol. 2002;23(4):183-189.

34. Mahmoud NN, Turpin RS, Yang G, Saunders WB. Impact of surgical site infections on length of stay and costs in selected colorectal procedures. Surg Infect. 2009;10(6):539-544.

35. Penel N, Lefebvre JL, Cazin JL, et al. Additional direct medical costs associated with mosocomial infections after head and neck cancer surgery: a hosptial-perspective analysis. Int J Oral Maxillofac Surg. 2008;37:135-139.

36. Jenney AW, Harrington GA, Russo PL, Spelman DW. Cost of surgical site infections following coronary artery bypass surgery. ANZ J Surg. 2001;71(11):662-664.

37. Olsen MA, Butler AM, Willers DM, Gross GA, Hamilton BH, Fraser VJ. Attributable costs of surgical site infection and endometritis after low transverse cesarean delivery. Infect Control Hosp Epidemiol. 2010; 31(3):276-282.

38. Blot SI, Depuydt P, Annemans L, et al. Clinical and economic outcomes in critically ill patients with nosocomial catheter-related bloodstream infections. Clin Infect Dis. 2005;41(11):1591-1598.

39. Orsi BG, Di Stefano L, Noah N. Hospital-acquired, laboratoryconfirmed bloodstream infection: Increased hospital stay and direct costs. Infect Control Hosp Epidemiol. 2002;23(4):190-197.

40. Pirson M, Dramaix M, Struelens M, Riley TV, Leclercq P. Costs associated with hospital-acquired bacteraemia in a Belgian hospital. $J$ Hosp Infect. 2005;59(1):33-40.

41. Pirson M, Leclercq P, Jackson T, Leclercq M, Garrino M, Sion C. Financial consequences of hospital-acquired bacteraemia in three Belgian hospitals in 2003 and 2004. J Hosp Infect. 2008;68(1):9-16.

42. Kilgore M, Brossette SE. Cost of bloodstream infections. Am J Infect Control. 2008;36:S172. e1-S172. e3.

43. Elward AM, Hollenbeak CS, Warren DK, Fraser VJ. Attributable cost of nosocomial primary bloodstream infection in pediatric intensive care unit patients. Pediatrics. 2005;115(4):868-872.

44. Payne NR, Carpenter JH, Badger GJ, Horbar JD, Rogowski J. Marginal increase in cost and excess length of stay associated with nosocomial bloodstream infections in surviving very low birth weight infants. Pediatrics. 2004;114(2):348-355.

45. Chu VH, Crosslin DR, Friedman JY, et al. Staphylococcus aureus bateremia in patients with prosthetic devices: costs and outcomes. $\mathrm{Am}$ J Med. 2005;118:1416. e19-1416. e24.

46. Adrie C,Alberti C, Chaix-Couturier C, et al. Epidemiology and economic evaluation of severe sepsis in France: age, severity, infection site, and palce of acquisition (community, hospital, or intensive care unit) as determinants of workload and cost. J Crit Care. 2005;20(1):46-58.

47. Brun-Buisson C, Roudot-Thoraval F, Girou E, Grenier-Sennelier C, Durand-Zaleski I. The costs of septic syndromes in the intensive care unit and influence of hospital-acquired sepsis. Intensive Care Med. 2003;29(9):1464-1471.

48. Festini F, Cocchi P, Mambretti D, et al. Nosocomial rotavirus gastroenteritis in pediatric patients: a multi-centre prospective cohort study. BMC Infect Dis. 2010;10:235-242.

49. Fruhwirth M, Berger K, Ehlken B, Moll-Schuler I, Brosl S, Mutz I. Economic impact of community- and nosocomially acquired rotavirus gastroenteritis in Austria. Pediatr Infect Dis J. 2001;20(2):184-188.

50. Piednoir E, Bessaci K, Bureau-Chalot F, et al. Economic impact of healthcare-associated rotavirus infection in a paediatric hospital. $J$ Hosp Infect. 2003;55(3):190-195. 
51. Tambyah PA, Knasinski V, Maki DG. The direct costs of nosocomial catheter-associated urinary tract infection in the era of managed care. Infect Control Hosp Epidemiol. 2002;23(1):27-31.

52. Morse BC, Boland BN, Blackhurst DW, Roettger RH. Analysis of centers for medicaid and medicare services 'Never events' in elderly patients undergoing bowel operations. Am Surg. 2010;76(8):841-845.

53. Rosenthal VD, Buzman S, Migone O, Safdar N. The attributable cost and length of hospital stay because of nosocomial pneumonia in intensive care units in 3 hospitals in Argentina: A prospective matched analysis. Am J Infect Control. 2005;33(3):157-161.

54. Dietrich ES, Demmler M, Schulgen G, et al. Nosocomial pneumonia: a cost-of-illness analysis. Infection. 2002;30(2):61-67.

55. Brilli RJ, Sparling KW, Lake MR, et al. The business case for preventing ventilator-associated pneumonia in pediatric intensive care unit patients. Jt Comm J Qual Saf. 2008;34(11):629-638.

56. Macartney KK, Gorelick MH, Manning ML, Hodinka RL, Bell LM. Nosocomial respiratory syncytial virus infections: the cost-effectiveness and cost-benefit of infection control. Pediatrics. 2000;106(3): 520-526.

57. Bou R, Lorente L, Aguilar A, et al. Hospital economic impact of an outbreak of Pseudomonas aeruginosa infections. J Hosp Infect. 2009; 71(2):138-142.

58. Fretz R, Schmid D, Jelovcan S, et al. An outbreak of norovirus gastroenteritis in an Austrian hospital, winter 2006-2007. Wien Klin Wochenschr. 2009;121(3-4):137-143.

59. Zingg W, Colombo C, Jucker T, Bossart W, Ruef C. Impact of an outbreak of norovirus infection on hospital resources. Infect Control Hosp Epidemiol. 2005;26(3):263-267.

60. Anil M, Helvaci M, Ozkalay N, et al. Salmonella typhimurium outbreak in a neonatal unit in Turkey. Indian J Pediatr. 2009;76(6): 629-633.

61. Baggett HC, Duchin JS, Shelton W, et al. Two nosocomial pertussis outbreaks and their associated costs - King County, Washington, 2004. Infect Control Hosp Epidemiol. 2007;28(5):537-543.

62. Spearing NM, Jensen A, McCall BJ, Neill AS, McCormack JG. Direct costs associated with a nosocomial outbreak of Salmonella infection: An ounce of prevention is worth a pound of cure. Am J Infect Control. 2000;28(1):54-57.

63. Wilson SJ, Knipe CJ, Zieger MJ, et al. Direct costs of multidrugresistance acinetobater baumannii in the burn unit of a public teaching hospital. Am J Infect Control. 2004;32:342-344.

64. Watters K, O'Dwyer TP, Rowley H. Cost and morbidity of the MRSA in head and neck cancer patients: what are the consequences? J Laryngol Otol. 2004;118(9):694-699.

65. Mauldin PD, Salgado CD, Durkalski VL, et al. Nosocomial infections due to mechicillin-resistant Staphylococcus aureus and vancomycinresistant Enterococcus: relationships with antibiotic use and cost drivers. Ann Pharmacother. 2008;42(3):317-326.

66. Vonberg RP, Reichardt C, Behnke M, Schwab F, Zindler S, Gastmeier P. Cost of nosocomial Clostridium difficile-associated diarrhoea. J Hosp Infect. 2008;70(1):15-20.

67. Puzniak LA, Gillespie KN, Leet T, Kollef M, Mundy LM. A cost-benefit analysis of gown use in controlling vancomycin-resistant enterococcus transmission: Is it worth the price? Infect Control Hosp Epidemiol. 2004;25(5):418-424.
68. Fuller RL, McCullough EC, Bao MZ, Averill RF. Estimating the costs of potentially preventable hospital acquired complications. Health Care Financ Rev. 2009;30(4):17-32.

69. Caprini JA, Botteman MF, Stephens JM, et al. Economic burden of longterm complications of deep vein thrombosis after total hip replacement surgery in the United States. Value Health. 2003;6(1):59-74.

70. MacDougall DA, Feliu AL, Boccuzzi SJ, Lin J. Economic burden of deep-vein thrombosis, pulmonary embolism, and post-thombotic syndrome. Am J Health-Syst Pharm. 2006;63(Suppl 6):S5-S15.

71. Nadkarni JB, Iyengar KP, Dussa C, Watve S, Vishwanath K. Orthopaedic injuries foloowing falls by hospital in-patients. Gerontology. 2005; 51:329-333.

72. Oliver D, Killick S, Even T, Willmott M. Do falls and falls-injuries in hospital indicate negligent care - and how big is the risk? A retrospective analysis of hte NHS litigation authority database of clinical negligence claims, resulting from falls in hospitals in England 1995 to 2006. Qual Saf Health Care. 2008;17:431-436.

73. Nurmi I, Luthje P. Incidence and costs of falls and fall injuries among elderly in institutional care. Scand J Prim Health Care. 2002;20(2): $118-122$.

74. Hill KD, Vu M, Walsh W. Falls in the acute hospital setting - impact on resource utilisation. Aus Health Rev. 2007;31(3):471-477.

75. Schmidek JM, Weeks WB. What do we know about financial returns on investments in patient safety? A literature review. Jt Comm J Qual Patient Saf. 2005;31(12):690-699.

76. Fukuda H, Imanaka Y. Assessment of transparency of cost estimates in economic evaluations of patient safety programmes. J Eval Clin Pract. 2009; $15(3): 451-459$.

77. Thomas EJ, Studdert DM, Newhouse JP, et al. Cost of medical injuries in Utah and Colorado. Inquiry. 1999;36(3):255-264.

78. Zhan C, Friedman B, Mosso A, Pronovost P. Medicare payment for selected adverse events: building the business case for investing in patient safety. Health Aff (Millwood). 2006;25(5):1386-1393.

79. Mello MM, Studdert DM, Thomas EJ, Yoon CS, Brennan TA. Who pays for medical errors? An analysis of adverse events costs, the medical liabililty system, and incentives for patient safety improvement. J Empir Leg Stud. 2011;4(4):835-860.

80. Graves N, Harbarth S, Beyersmann J, Barnett A, Halton K, Copper B. Estimating the cost of health care-associated infecitons: mind your p's and q's. Clin Infect Dis. 2010;50(7):1017-1021.

81. Barnett AG, Batra R, Graves N, Edgeworth J, Robotham J, Cooper B. Using a longitudinal model to estimate the effect of methicillin-resistant Staphylococcus aureus infection on length of stay in an intensive care unit. Am J Epidemiol. 2009;170(9):1186-1194.

82. Graves N, Weinhold D, Roberts JA. Correcting for bias when estimating the cost of hospital-acquired infection: an analysis of lower repiratory tract infections in non-surgical patients. Health Econ. 2005;14(7): 755-761.

83. Beyersmann J, Kneib T, Schumacher M, Gastmeier P. Nosocomial infection, length of stay, and time-dependent bias. Infect Control Hosp Epidemiol. 2009;30(3):273-276.

84. Shreve J, Van Den Bos J, Gray T, Halford M, Rustagi K, Ziemkiewicz E. The economic measurement of medical errors. 2010. Available from: http://www.soa.org/files/pdf/research-econ-measurement.pdf. Accessed September 30, 2011.

Drug, Healthcare and Patient Safety

\section{Publish your work in this journal}

Drug, Healthcare and Patient Safety is an international, peer-reviewed open-access journal exploring patient safety issues in the healthcare continuum from diagnostic and screening interventions through to treatment, drug therapy and surgery. The journal is characterized by the rapid reporting of reviews, original research, clinical, epidemiological and

post-marketing surveillance studies, risk management, health literacy and educational programs across all areas of healthcare delivery. The manuscript management system is completely online and includes a very quick and fair peer-review system. Visit http://www.dovepress.com/ testimonials.php to read real quotes from published authors. 